\title{
Martingales and Super-Martingales Relative to a Convex Set of Equivalent Measures
}

\author{
Nicholas S. Gonchar \\ Bogolyubov Institute for Theoretical Physics of NAS, Kiev, Ukraine \\ Email: mhonchar@i.ua
}

How to cite this paper: Gonchar, N.S. (2018) Martingales and Super-Martingales Relative to a Convex Set of Equivalent Measures. Advances in Pure Mathematics, 8, 428-462.

https://doi.org/10.4236/apm.2018.84025

Received: December 17, 2017

Accepted: April 21, 2018

Published: April 24, 2018

Copyright $\odot 2018$ by author and Scientific Research Publishing Inc. This work is licensed under the Creative Commons Attribution International License (CC BY 4.0).

http://creativecommons.org/licenses/by/4.0/

(c) (i) Open Access

\begin{abstract}
In the paper, the martingales and super-martingales relative to a convex set of equivalent measures are systematically studied. The notion of local regular super-martingale relative to a convex set of equivalent measures is introduced and the necessary and sufficient conditions of the local regularity of it in the discrete case are founded. The description of all local regular super-martingales relative to a convex set of equivalent measures is presented. The notion of the complete set of equivalent measures is introduced. We prove that every bounded in some sense super-martingale relative to the complete set of equivalent measures is local regular. A new definition of the fair price of contingent claim in an incomplete market is given and the formula for the fair price of Standard Option of European type is found. The proved Theorems are the generalization of the famous Doob decomposition for super-martingale onto the case of super-martingales relative to a convex set of equivalent measures.
\end{abstract}

\section{Keywords}

Random Process, Convex Set of Equivalent Measures, Optional Doob Decomposition, Local Regular Super-Martingale, Martingale, Fair Price of Contingent Claim

\section{Introduction}

In the paper, a new method of investigation of martingales and super-martingales relative to a convex set of equivalent measures is developed. A new proof that the essential supremum over the set of regular martingales, generated by a certain nonnegative random value and a convex set of equivalent measures, is a super-martingale with respect to this set of measures, is given.

A notion of local regular super-martingale is introduced and the necessary and sufficient conditions are found under that the above defined super-martingales 
are local regular ones. The last fact allowed us to describe the local regular super-martingales. It is proved that the existence of a nontrivial martingale relative to a convex set of equivalent measures, generally speaking, does not guarantee for a nonnegative super-martingale to be a local regular one.

An important notion of the complete convex set of equivalent measures is introduced. It is proved that any super-martingale relative to the complete convex set of equivalent measures on a measurable space with the finite set of elementary events is a local regular one. The notion of the complete convex set of equivalent measures is generalized onto an arbitrary space of elementary events. It is proved that the nonnegative and the majorized from below super-martingales are local regular ones.

The definition of the fair price of contingent claim is introduced. The sufficient conditions of the existence of the fair price of contingent claim are presented. The conditions that the introduced notion coincides with classical one are given.

All these notions are used in the case as the convex set of equivalent measures is a set of equivalent martingale measures for the evolution of both risk and non-risk assets. The formula for the fair price of Standard Contract with Option of European type in an incomplete market is found.

The notion of the complete convex set of equivalent measures permits us to give a new proof of the optional decomposition for a nonnegative super-martingale. This proof does not use the no-arbitrage arguments and the measurable choice [1] [2] [3] [4].

First, the optional decomposition for diffusion processes super-martingale was opened by El Karoui N. and Quenez M. C. [5]. After that, Kramkov D. O. and Follmer H. [1] [2] proved the optional decomposition for the nonnegative bounded super-martingales. Folmer H. and Kabanov Yu. M. [3] [4] proved analogous result for an arbitrary super-martingale. Recently, Bouchard B. and Nutz M. [6] considered a class of discrete models and proved the necessary and sufficient conditions for the validity of the optional decomposition.

The optional decomposition for super-martingales plays the fundamental role for the risk assessment in incomplete markets [1] [2] [5] [7] [8] [9] [10] [11]. Considered in the paper problem is a generalization of the corresponding one that appeared in mathematical finance about the optional decomposition for a super-martingale and which is related with the construction of the superhedge strategy in incomplete financial markets.

Our statement of the problem unlike the above-mentioned one and it is more general: a super-martingale relative to a convex set of equivalent measures is given and it is necessary to find the conditions for the super-martingale and the set of measures under that the optional decomposition exists.

The generality of our statement of the problem is that we do not require that the considered set of measures was generated by the random process that is a local martingale as it is done in the papers [1] [4] [5] [6] and that is important 
for the proof of the optional decomposition in these papers.

\section{Local Regular Super-Martingales Relative to a Convex Set of Equivalent Measures}

We assume that on a measurable space $\{\Omega, \mathcal{F}\}$ a filtration $\mathcal{F}_{m} \subset \mathcal{F}_{m+1} \subset \mathcal{F}, m=\overline{0, \infty}$, and a family of convex set of equivalent measures $M$ on $\mathcal{F}$ are given. Further, we assume that $\mathcal{F}_{0}=\{\varnothing, \Omega\}$ and the $\sigma$-algebra $\mathcal{F}=\sigma\left(\bigvee_{n=1}^{\infty} \mathcal{F}_{n}\right)$ is a minimal $\sigma$-algebra generated by the algebra $\bigvee_{n=1}^{\infty} \mathcal{F}_{n}$. A random process $\psi=\left\{\psi_{m}\right\}_{m=0}^{\infty}$ is said to be adapted one relative to the filtration $\left\{\mathcal{F}_{m}\right\}_{m=0}^{\infty}$ if $\psi_{m}$ is a $\mathcal{F}_{m}$ measurable random value, $m=\overline{0, \infty}$.

Definition 1. An adapted random process $f=\left\{f_{m}\right\}_{m=0}^{\infty}$ is said to be a super-martingale relative to the filtration $\mathcal{F}_{m}, m=\overline{0, \infty}$, and the convex family of equivalent measures $M$ if $E^{P}\left|f_{m}\right|<\infty, m=\overline{1, \infty}, P \in M$, and the inequalities

$$
E^{P}\left\{f_{m} \mid \mathcal{F}_{k}\right\} \leq f_{k}, 0 \leq k \leq m, m=\overline{1, \infty}, P \in M,
$$

are valid.

Further, for an adapted process $f$ we use both the denotation $\left\{f_{m}, \mathcal{F}_{m}\right\}_{m=0}^{\infty}$ and the denotation $\left\{f_{m}\right\}_{m=0}^{\infty}$.

Definition 2. A super-martingale $\left\{f_{m}, \mathcal{F}_{m}\right\}_{m=0}^{\infty}$ relative to a convex set of equivalent measures $M$ is a local regular one if $\sup _{P \in M} E^{P}\left|f_{m}\right|<\infty, m=\overline{1, \infty}$, and there exists an adapted nonnegative increasing random process $\left\{g_{m}, \mathcal{F}_{m}\right\}_{m=0}^{\infty}, g_{0}=0, \sup _{P \in M} E^{P}\left|g_{m}\right|<\infty, m=\overline{1, \infty}$, such that $\left\{f_{m}+g_{m}, \mathcal{F}_{m}\right\}_{m=0}^{\infty}$ is a martingale relative to every measure from $M$.

The next elementary Theorem 1 will be very useful later.

Theorem 1. Let a super-martingale $\left\{f_{m}, \mathcal{F}_{m}\right\}_{m=0}^{\infty}$, relative to a convex set of equivalent measures $M$ be such that $\sup E^{P}\left|f_{m}\right|<\infty, m=\overline{1, \infty}$. The necessary and sufficient condition for it to be a focal regular one is the existence of an adapted nonnegative random process $\left\{\bar{g}_{m}^{0}, \mathcal{F}_{m}\right\}_{m=0}^{\infty}, \sup _{P \in M} E^{P}\left|\bar{g}_{m}^{0}\right|<\infty, m=\overline{1, \infty}$, such that

$$
f_{m-1}-E^{P}\left\{f_{m} \mid \mathcal{F}_{m-1}\right\}=E^{P}\left\{\bar{g}_{m}^{0} \mid \mathcal{F}_{m-1}\right\}, m=\overline{1, \infty}, P \in M .
$$

Proof. Necessity. If $\left\{f_{m}, \mathcal{F}_{m}\right\}_{m=0}^{\infty}$ is a local regular super-martingale, then there exist a martingale $\left\{\bar{M}_{m}, \mathcal{F}_{m}\right\}_{m=0}^{\infty}$ and a non-decreasing nonnegative random process $\left\{g_{m}, \mathcal{F}_{m}\right\}_{m=0}^{\infty}, g_{0}=0$, such that

$$
f_{m}=\bar{M}_{m}-g_{m}, m=\overline{1, \infty} \text {. }
$$

From here we obtain the equalities

$$
\begin{aligned}
& E^{P}\left\{f_{m-1}-f_{m} \mid \mathcal{F}_{m-1}\right\} \\
& =E^{P}\left\{g_{m}-g_{m-1} \mid \mathcal{F}_{m-1}\right\}=E^{P}\left\{\bar{g}_{m}^{0} \mid \mathcal{F}_{m-1}\right\}, m=\overline{1, \infty}, P \in M,
\end{aligned}
$$


where we introduced the denotation $\bar{g}_{m}^{0}=g_{m}-g_{m-1} \geq 0$. It is evident that $E^{P} \bar{g}_{m}^{0} \leq \sup _{P \in M} E^{P} g_{m}+\sup _{P \in M} E^{P} g_{m-1}<\infty$.

Sufficiency. Suppose that there exists an adapted nonnegative random process $\bar{g}^{0}=\left\{\bar{g}_{m}^{0}\right\}_{m=0}^{\infty}, \bar{g}_{0}^{0}=0, E^{P} \bar{g}_{m}^{0}<\infty, m=\overline{1, \infty}$, such that the equalities (2) hold. Let us consider the random process $\left\{\bar{M}_{m}, \mathcal{F}_{m}\right\}_{m=0}^{\infty}$, where

$$
\bar{M}_{0}=f_{0}, \bar{M}_{m}=f_{m}+\sum_{i=1}^{m} \bar{g}_{m}^{0}, m=\overline{1, \infty} .
$$

It is evident that $E^{P}\left|\bar{M}_{m}\right|<\infty$ and

$$
E^{P}\left\{\bar{M}_{m-1}-\bar{M}_{m} \mid \mathcal{F}_{m-1}\right\}=E^{P}\left\{f_{m-1}-f_{m}-\bar{g}_{m}^{0} \mid \mathcal{F}_{m-1}\right\}=0 .
$$

Theorem 1 is proved.

Lemma 1. Any super-martingale $\left\{f_{m}, \mathcal{F}_{m}\right\}_{m=0}^{\infty}$ relative to a family of measures $M$ for which there hold equalities $E^{P} f_{m}=f_{0}, m=\overline{1, \infty}, P \in M$ is a martingale with respect to this family of measures and the filtration $\mathcal{F}_{m}, m=\overline{1, \infty}$.

Proof. The proof of Lemma 1 see [12].

\section{Description of Local Regular Super-Martingales Relative to a Convex Set of Equivalent Measures Generated by the Finite Set of Equivalent Measures}

Below, we describe the local regular super-martingales relative to a convex set of equivalent measures $M$ generated by the finite set of equivalent measures. For this we need some auxiliary statements.

Lemma 2. On a measurable space $\{\Omega, \mathcal{F}\}$ with filtration $\mathcal{F}_{m}$ on it, let $G$ be a sub $\sigma$-algebra of the $\sigma$-algebra $\mathcal{F}$ and let $f_{s}, s \in S$ be a finite family of nonnegative bounded random values. Then for every measure $P$ from $M$.

$$
E^{P}\left\{\max _{s \in S} f_{s} \mid G\right\} \geq \max _{s \in S} E^{P}\left\{f_{s} \mid G\right\}, P \in M .
$$

Proof. We have the inequalities

$$
\max _{s \in S} f_{s} \geq f_{t}, t \in S .
$$

Therefore,

$$
E^{P}\left\{\max _{s \in S} f_{s} \mid G\right\} \geq E^{P}\left\{f_{t} \mid G\right\}, t \in S, P \in M .
$$

The last implies

$$
E^{P}\left\{\max _{s \in S} f_{s} \mid G\right\} \geq \max _{s \in S} E^{P}\left\{f_{s} \mid G\right\}, P \in M .
$$

In the next Lemma, we present the formula for calculation of the conditional expectation relative to another measure from $M$.

Lemma 3. On a measurable space $\{\Omega, \mathcal{F}\}$ with a filtration $\mathcal{F}_{n}$ on it, let $M$ be a convex set of equivalent measures and let $\xi$ be a bounded random value. Then the following formulas

$$
E^{P_{1}}\left\{\xi \mid \mathcal{F}_{n}\right\}=E^{P_{2}}\left\{\xi \varphi_{n}^{P_{1}} \mid \mathcal{F}_{n}\right\}, \quad n=\overline{1, \infty},
$$


are valid, where

$$
\varphi_{n}^{P_{1}}=\frac{\mathrm{d} P_{1}}{\mathrm{~d} P_{2}}\left[E^{P_{2}}\left\{\frac{\mathrm{d} P_{1}}{\mathrm{~d} P_{2}} \mid \mathcal{F}_{n}\right\}\right]^{-1}, P_{1}, P_{2} \in M .
$$

Proof. The proof of Lemma 3 is evident.

Let $P_{1}, \cdots, P_{k}$ be a family of equivalent measures on a measurable space $\{\Omega, \mathcal{F}\}$ and let us introduce the denotation $M$ for a convex set of equivalent measures

$$
M=\left\{Q, Q=\sum_{i=1}^{k} \alpha_{i} P_{i}, \alpha_{i} \geq 0, i=\overline{1, k}, \sum_{i=1}^{k} \alpha_{i}=1\right\} .
$$

Lemma 4. If $\xi$ is an integrable random value relative to the set of equivalent measures $P_{1}, \cdots, P_{k}$, then the formula

$$
\operatorname{ess} \sup _{Q \in M} E^{Q}\left\{\xi \mid \mathcal{F}_{n}\right\}=\max _{1<i<k} E^{P_{i}}\left\{\xi \mid \mathcal{F}_{n}\right\}
$$

is valid almost everywhere relative to the measure $P_{1}$.

Proof. The definition of esssup for non countable family of random variables see [13]. Using the formula

$$
E^{Q}\left\{\xi \mid F_{n}\right\}=\frac{\sum_{i=1}^{k} \alpha_{i} E^{P_{1}}\left\{\varphi_{i} \mid \mathcal{F}_{n}\right\} E^{P_{i}}\left\{\xi \mid \mathcal{F}_{n}\right\}}{\sum_{i=1}^{k} \alpha_{i} E^{P_{1}}\left\{\varphi_{i} \mid \mathcal{F}_{n}\right\}}, Q \in M,
$$

where $\varphi_{i}=\frac{\mathrm{d} P_{i}}{\mathrm{~d} P_{1}}, Q=\sum_{i=1}^{k} \alpha_{i} P_{i}$, we obtain the inequality

$$
E^{Q}\left\{\xi \mid \mathcal{F}_{n}\right\} \leq \max _{1 \leq i \leq k} E^{P_{i}}\left\{\xi \mid \mathcal{F}_{n}\right\}, Q \in M,
$$

or,

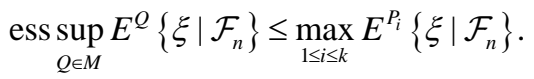

On the other side [13],

$$
E^{P_{i}}\left\{\xi \mid \mathcal{F}_{n}\right\} \leq \operatorname{ess} \sup _{Q \in M} E^{Q}\left\{\xi \mid \mathcal{F}_{n}\right\}, i=\overline{1, k} .
$$

Therefore,

$$
\max _{1 \leq i \leq k} E^{P_{i}}\left\{\xi \mid \mathcal{F}_{n}\right\} \leq \operatorname{ess} \sup _{Q \in M} E^{Q}\left\{\xi \mid \mathcal{F}_{n}\right\} .
$$

Lemma 4 is proved.

Lemma 5. On a measurable space $\{\Omega, \mathcal{F}\}$ with a filtration $\mathcal{F}_{n}$ on it, let $\xi$ be a nonnegative bounded random value. If $\frac{\mathrm{d} P_{i}}{\mathrm{~d} P_{l}}, i, l=\overline{1, k}$ are $\mathcal{F}_{1}$ measurable and $P_{1}\left(\frac{\mathrm{d} P_{i}}{\mathrm{~d} P_{l}}>0\right)=1, i, l=1, k$, then the inequalities

$$
E^{P_{l}}\left\{\max _{1 \leq i \leq k} E^{P_{i}}\left\{\xi \mid \mathcal{F}_{n}\right\} \mid \mathcal{F}_{m}\right\} \leq \max _{1 \leq i \leq k} E^{P_{i}}\left\{\xi \mid \mathcal{F}_{m}\right\}, l=\overline{1, k}, n>m,
$$


are valid.

Proof. From Lemma 3 and Lemma 5 conditions relative to the density of one measure with respect to another, we have

$$
\max _{1<i \leq k} E^{P_{i}}\left\{\xi \mid \mathcal{F}_{n}\right\}=E^{P_{i}}\left\{\xi \mid \mathcal{F}_{n}\right\}, l=\overline{1, k}
$$

From the equality (21) we obtain the inequality

$$
E^{P_{l}}\left\{\max _{1 \leq i \leq k} E^{P_{i}}\left\{\xi \mid \mathcal{F}_{n}\right\} \mid \mathcal{F}_{m}\right\} \leq \max _{1 \leq i \leq k} E^{P_{i}}\left\{\xi \mid \mathcal{F}_{m}\right\}, l=\overline{1, k} .
$$

Lemma 5 is proved.

In this section, we assume that the conditions of Lemma 5 relative to the density of one measure with respect to another are true.

Lemma 6. On a measurable space $\{\Omega, \mathcal{F}\}$ with a filtration $\mathcal{F}_{n}$ on it, let $\xi$ be a nonnegative random value which is integrable relative to the set of equivalent measures $P_{1}, \cdots, P_{k}$. Then the inequalities

$$
E^{Q}\left\{\max _{1 \leq i \leq k} E^{P_{i}}\left\{\xi \mid \mathcal{F}_{n}\right\} \mid \mathcal{F}_{m}\right\} \leq \max _{1 \leq i \leq k} E^{P_{i}}\left\{\xi \mid \mathcal{F}_{m}\right\}, n>m, Q \in M,
$$

are valid.

Proof. Using Lemma 5 inequalities for the nonnegative bounded $\xi$ and the formula

$$
E^{Q}\left\{\Phi \mid \mathcal{F}_{m}\right\}=\frac{\sum_{i=1}^{k} \alpha_{i} E^{P_{1}}\left\{\varphi_{i} \mid \mathcal{F}_{m}\right\} E^{P_{i}}\left\{\Phi \mid \mathcal{F}_{m}\right\}}{\sum_{i=1}^{k} \alpha_{i} E^{P_{1}}\left\{\varphi_{i} \mid \mathcal{F}_{m}\right\}}, Q \in M,
$$

where $\Phi=\max _{1 \leq i \leq k} E^{P_{i}}\left\{\xi \mid \mathcal{F}_{n}\right\}, \varphi_{i}=\frac{\mathrm{d} P_{i}}{\mathrm{~d} P_{1}}, i=\overline{1, k}$, we prove Lemma 6 inequalities.

Let us consider the case, as $\max _{1 \leq i \leq k} E^{P_{i}} \xi<\infty$. Let $\xi_{s}, s=\overline{1, \infty}$ be a sequence of bounded random values converging to $\xi$ monotonuosly. Then

$$
E^{Q}\left\{\max _{1 \leq i \leq k} E^{P_{i}}\left\{\xi_{s} \mid \mathcal{F}_{n}\right\} \mid \mathcal{F}_{m}\right\} \leq \max _{1 \leq i \leq k} E^{Q}\left\{\xi_{s} \mid \mathcal{F}_{m}\right\}, l=\overline{1, k} .
$$

Due to the monotony convergence of $\xi_{s}$ to $\xi$, as $s \rightarrow \infty$, we can pass to the limit under the conditional expectations on the left and right sides in the inequalities (25) that proves Lemma 6.

Lemma 7. On a measurable space $\{\Omega, \mathcal{F}\}$ with filtration $\mathcal{F}_{n}$ on it, for every nonnegative integrable random value $\xi$ relative to a set of equivalent measures $\left\{P_{1}, \cdots, P_{k}\right\}$ the inequalities

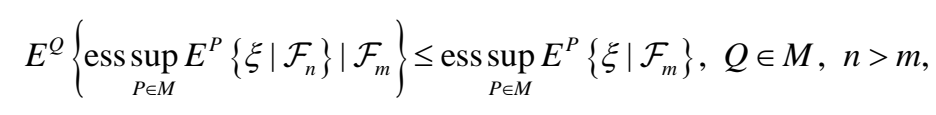

are valid.

Lemma 7 is a consequence of Lemma 6.

Lemma 8. On a measurable space $\{\Omega, \mathcal{F}\}$ with a filtration $\mathcal{F}_{m}$ on it, let $\xi$ be a nonnegative integrable random value with respect to a set of equivalent measures $\left\{P_{1}, \cdots, P_{k}\right\}$ and such that 


$$
E^{P_{i}} \xi=M_{0}, i=\overline{1, k},
$$

then the random process $\left\{M_{m}=\underset{P \in M}{\operatorname{ess} \sup } E^{P}\left\{\xi \mid \mathcal{F}_{m}\right\}, \mathcal{F}_{m}\right\}_{m=0}^{\infty}$ is a martingale relative to a convex set of equivalent measures $M$.

Proof. Due to Lemma 7, a random process

$$
\begin{gathered}
\left\{M_{m}=\underset{P \in M}{\operatorname{esssup}} E^{P}\left\{\xi \mid \mathcal{F}_{m}\right\}, \mathcal{F}_{m}\right\}_{m=0}^{\infty} \text { is a super-martingale, that is, } \\
E^{P}\left\{M_{m} \mid \mathcal{F}_{m-1}\right\} \leq M_{m-1}, \quad m=\overline{1, \infty}, P \in M .
\end{gathered}
$$

Or, $E^{P} M_{m} \leq M_{0}$. From the other side,

$$
E^{P_{s}}\left[\max _{1 \leq i \leq k} E^{P_{i}}\left\{\xi \mid \mathcal{F}_{m}\right\}\right] \geq \max _{1 \leq i \leq k} E^{P_{s}} E^{P_{i}}\left\{\xi \mid \mathcal{F}_{m}\right\} \geq M_{0}, s=\overline{1, k} .
$$

The above inequalities imply $E^{P_{s}} M_{m}=M_{0}, m=\overline{1, \infty}, s=\overline{1, k}$. The last equalities lead to the equalities $E^{P} M_{m}=M_{0}, m=\overline{1, \infty}, P \in M$. The fact that $M_{m}$ is a super-martingale relative to the set of measures $M$ and the above equalities prove Lemma 8, since the Lemma 1 conditions are valid.

In the next Theorem we denote $\mathcal{F}=\sigma\left(\bigvee_{i=1}^{\infty} \mathcal{F}_{i}\right)$ the minimal $\sigma$-algebra generated by the algebra $\bigvee_{i=1}^{\infty} \mathcal{F}_{i}$.

Theorem 2. Let $\{\Omega, \mathcal{F}\}$ be a measurable space with a filtration $\mathcal{F}_{m}$ on it and let $\xi$ be a nonnegative integrable random value with respect to a set of equivalent measures $P_{1}, \cdots, P_{k}$. The necessary and sufficient conditions of the local regularity of the super-martingale $\left\{f_{m}, \mathcal{F}_{m}\right\}_{m=0}^{\infty}$, where

$$
f_{m}=\operatorname{ess} \sup _{P \in M} E^{P}\left\{\xi \mid \mathcal{F}_{m}\right\}, m=\overline{1, \infty}, \max _{1 \leq i \leq k} E^{P_{i}} \xi<\infty,
$$

is its uniform integrability relative to the set of measure $P_{1}, \cdots, P_{k}$ and the fulfillment of the equalities

$$
E^{P_{i}} \xi=f_{0}, i=\overline{1, k} .
$$

Proof. The necessity. Let $\left\{f_{m}, \mathcal{F}_{m}\right\}_{m=0}^{\infty}$ be a local regular super-martingale. Then

$$
f_{n}=M_{n}-g_{n}, n=\overline{0, \infty}, g_{0}=0, \quad f_{0}=E^{P_{i}} M_{n}, i=\overline{1, k} .
$$

From here we obtain $E^{P_{i}} g_{n} \leq f_{0}, i=\overline{1, k}$. Due to the uniform integrability of $f_{n}$ and $g_{n}$ we obtain

$$
E^{P_{i}}\left(f_{\infty}+g_{\infty}\right)=f_{0}, i=\overline{1, k},
$$

where $f_{\infty}=\xi, g_{\infty}=\lim _{n \rightarrow \infty} g_{n}$, since $\mathcal{F}=\sigma\left(\bigvee_{i=1}^{\infty} \mathcal{F}_{i}\right)$. But $f_{0}=\max _{1 \leq i \leq k} E^{P_{i}} \xi=E^{P_{i_{0}}} \xi$. From (33) we have $E^{P^{P_{0}}} g_{\infty}=0$. The last equality gives $g_{\infty}=0$, or

$$
E^{P_{i}} \xi=E^{P_{i 0}} \xi, i=\overline{1, k}
$$

The sufficiency. If the conditions of Theorem 2 are satisfied, then $\left\{\bar{M}_{m}, \mathcal{F}_{m}\right\}_{m=0}^{\infty}$ is a martingale, where $\bar{M}_{m}=\sup _{P \in M} E^{P}\left\{\xi \mid \mathcal{F}_{m}\right\}$. The last implies the 
local regularity of $\left\{f_{m}, \mathcal{F}_{m}\right\}_{m=0}^{\infty}$. Theorem 2 is proved.

\section{Description of Local Regular Super-Martingales Relative to an Arbitrary Convex Set of Equivalent Measures}

Below, in the paper we assume that an arbitrary convex set of equivalent measures $M$ on a measurable space $\{\Omega, \mathcal{F}\}$ and a filtration $\mathcal{F}_{n}$ on it satisfies the conditions: the density $\frac{\mathrm{d} P}{\mathrm{~d} Q}$ is $\mathcal{F}_{1}$ measurable one and $P_{0}\left(\frac{\mathrm{d} P}{\mathrm{~d} Q}>0\right)=1$ for all $P, Q \in M$, where the fixed measure $P_{0} \in M$. Such a class of equivalent measures is sufficiently wide. It contains the class of equivalent martingale measures generated by a local martingale.

Introduce into consideration a set $A_{0}$ of all integrable nonnegative random values $\xi$ relative to a convex set of equivalent measures $M$ satisfying conditions

$$
E^{P} \xi=1, P \in M .
$$

It is evident that the set $A_{0}$ is not empty, since contains the random value $\xi=1$. More interesting case is as $A_{0}$ contains more then one element.

Lemma 9. On a measurable space $\{\Omega, \mathcal{F}\}$ and a filtration $\mathcal{F}_{n}$ on it, let $M$ be an arbitrary convex set of equivalent measures. If the nonnegative random value $\xi$ is such that $\sup _{P \in M} E^{P} \xi<\infty$, then $\left\{f_{m}=\underset{P \in M}{\operatorname{esssup}} E^{P}\left\{\xi \mid \mathcal{F}_{m}\right\}, \mathcal{F}_{m}\right\}_{m=0}^{\infty}$ is a super-martingale relative to the convex set of equivalent measures $M$.

Proof. From the definition of esssup [13], for every $\underset{P \in M}{\operatorname{esssup}} E^{P}\left\{\xi \mid \mathcal{F}_{m}\right\}$ there exists a countable set $D_{m}$ such that

$$
\operatorname{ess} \sup _{P \in M} E^{P}\left\{\xi \mid \mathcal{F}_{m}\right\}=\sup _{P \in D_{m}} E^{P}\left\{\xi \mid \mathcal{F}_{m}\right\}, \quad m=\overline{0, \infty} \text {. }
$$

The set $D=\bigcup_{m=0}^{\infty} D_{m}$ is also countable one and the equality

$$
\underset{P \in M}{\operatorname{ess} \sup _{P}} E^{P}\left\{\xi \mid \mathcal{F}_{m}\right\}=\sup _{P \in D} E^{P}\left\{\xi \mid \mathcal{F}_{m}\right\}
$$

is true. Really, since

$$
\sup _{P \in D} E^{P}\left\{\xi \mid \mathcal{F}_{m}\right\} \geq \sup _{P \in D_{M}} E^{P}\left\{\xi \mid \mathcal{F}_{m}\right\}=\operatorname{ess} \sup _{P \in M} E^{P}\left\{\xi \mid \mathcal{F}_{m}\right\} .
$$

From the other side,

$$
\underset{P \in M}{\operatorname{ess} \sup E^{P}}\left\{\xi \mid \mathcal{F}_{m}\right\} \geq E^{Q}\left\{\xi \mid \mathcal{F}_{m}\right\}, Q \in M .
$$

The last gives

$$
\underset{P \in M}{\operatorname{ess} \sup } E^{P}\left\{\xi \mid \mathcal{F}_{m}\right\} \geq \sup _{P \in D} E^{P}\left\{\xi \mid \mathcal{F}_{m}\right\} .
$$

The inequalities (38), (40) prove the needed statement. So, for all $m$ we can choose the common set $D$. Let $D=\left\{\bar{P}_{1}, \cdots, \bar{P}_{n}, \cdots\right\}$. Due to Lemma 7 , for every $Q \in \bar{M}_{k}$, we have 


$$
E^{Q}\left\{\max _{1 \leq i \leq k} E^{\bar{P}_{i}}\left\{\xi \mid \mathcal{F}_{n}\right\} \mid \mathcal{F}_{m}\right\} \leq \max _{1 \leq i \leq k} E^{\bar{P}_{i}}\left\{\xi \mid \mathcal{F}_{m}\right\}, n>m, Q \in \bar{M}_{k}
$$

where

$$
\bar{M}_{k}=\left\{P \in M, P=\sum_{i=1}^{k} \alpha_{i} \bar{P}_{i}, \alpha_{i} \geq 0, \sum_{i=1}^{k} \alpha_{i}=1\right\} .
$$

It is evident that $\max _{1<i \leq k} E^{\bar{P}_{i}}\left\{\xi \mid \mathcal{F}_{n}\right\}$ tends to $\sup _{P \in D} E^{P}\left\{\xi \mid \mathcal{F}_{n}\right\}$ monotonously increasing, as $k \rightarrow \infty$. Fixing $Q \in \bar{M}_{k} \subset \bar{M}_{k+1}$ and tending $k$ to the infinity in the inequalities (41), we obtain

$$
E^{Q}\left\{\sup _{P \in D} E^{P}\left\{\xi \mid \mathcal{F}_{n}\right\} \mid \mathcal{F}_{m}\right\} \leq \sup _{P \in D} E\left\{\xi \mid \mathcal{F}_{m}\right\}, n>m, Q \in \bar{M}_{k} .
$$

The last inequalities implies that for every measure $Q$, belonging to the convex span, constructed on the set $D,\left\{f_{m}=\underset{P \in M}{\operatorname{esssup}} E^{P}\left\{\xi \mid \mathcal{F}_{m}\right\}, \mathcal{F}_{m}\right\}_{m=0}^{\infty}$ is a super-martingale relative to the convex set of equivalent measures, generated by the set $D$. Now, if a measure $Q_{0}$ does not belong to the convex span, constructed on the set $D$, then we can add it to the set $D$ and repeat the proof made above. As

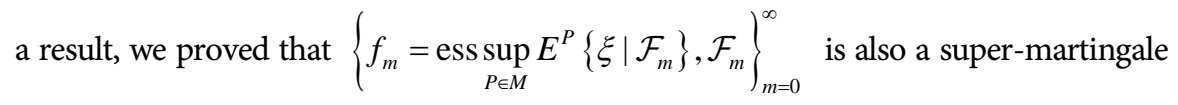
relative to the measure $Q_{0}$. Zorn Lemma [14] complete the proof of Lemma 9.

Theorem 3. On a measurable space $\{\Omega, \mathcal{F}\}$ and a filtration $\mathcal{F}_{n}$ on it, let $M$ be an arbitrary convex set of equivalent measures. For a random value $\xi \in A_{0}$, the random process $\left\{E^{P}\left\{\xi \mid \mathcal{F}_{m}\right\}, \mathcal{F}_{m}\right\}_{m=0}^{\infty}, P \in M$ is a local regular martingale relative to the convex set of equivalent measures $M$.

Proof. Let $P_{1}, \cdots, P_{n}$ be a certain subset of measures from $M$. Denote $M_{n}$ a convex set of equivalent measures

$$
M_{n}=\left\{P \in M, P=\sum_{i=1}^{n} \alpha_{i} P_{i}, \alpha_{i} \geq 0, i=\overline{1, n}, \sum_{i=1}^{n} \alpha_{i}=1\right\} .
$$

Due to Lemma $8,\left\{\bar{M}_{m}, \mathcal{F}_{m}\right\}_{m=0}^{\infty}$ is a martingale relative to the set of measures $M_{n}$, where $\bar{M}_{m}=$ ess $\sup _{P \in M_{n}} E^{P}\left\{\xi \mid \mathcal{F}_{m}\right\}, \xi \in A_{0}$. Let us consider an arbitrary measure $P_{0} \in M$ and let

$$
M_{n}^{P_{0}}=\left\{P \in M, P=\sum_{i=0}^{n} \alpha_{i} P_{i}, \alpha_{i} \geq 0, i=\overline{0, n}, \sum_{i=0}^{n} \alpha_{i}=1\right\} .
$$

Then $\left\{\bar{M}_{m}^{P_{0}}, \mathcal{F}_{m}\right\}_{m=0}^{\infty}$, where $\bar{M}_{m}^{P_{0}}=$ ess $\sup _{P \in M_{n}^{P_{0}}} E^{P}\left\{\xi \mid \mathcal{F}_{m}\right\}$, is a martingale relative to the set of measures $M_{n}^{P_{0}}$. It is evident that

$$
\bar{M}_{m} \leq \bar{M}_{m}^{P_{0}}, m=\overline{0, \infty} .
$$

Since $E^{P} \bar{M}_{m}=E^{P} \bar{M}_{m}^{P_{0}}=1, m=\overline{0, \infty}, P \in M_{n}$, the inequalities (46) give $\bar{M}_{m}=\bar{M}_{m}^{P_{0}}$. Analogously, $E^{P_{0}}\left\{\xi \mid \mathcal{F}_{m}\right\} \leq \bar{M}_{m}^{P_{0}}$. From the equalities $E^{P_{0}} E^{P_{0}}\left\{\xi \mid \mathcal{F}_{m}\right\}=E^{P_{0}} \bar{M}_{m}^{P_{0}}=1$ we obtain $E^{P_{0}}\left\{\xi \mid \mathcal{F}_{m}\right\}=\bar{M}_{m}^{P_{0}}=\bar{M}_{m}$. Since the 
measure $P_{0}$ is an arbitrary one it implies that $\left\{E^{P}\left\{\xi \mid \mathcal{F}_{m}\right\}, \mathcal{F}_{m}\right\}_{m=0}^{\infty}$ is a martingale relative to all measures from $M$. Due to Theorem 1 , it is a local regular super-martingale with the random process $\bar{g}_{m}^{0}=0, m=\overline{0, \infty}$. Theorem 3 is proved.

Theorem 4. On a measurable space $\{\Omega, \mathcal{F}\}$ and a filtration $\mathcal{F}_{n}$ on it, let $M$ be an arbitrary convex set of equivalent measures. If $\left\{f_{m}, \mathcal{F}_{m}\right\}_{m=0}^{\infty}$ is an adapted random process satisfying conditions

$$
f_{m} \leq f_{m-1}, E^{P} \xi\left|f_{m}\right|<\infty, P \in M, m=\overline{1, \infty}, \xi \in A_{0},
$$

then the random process

$$
\left\{f_{m} E^{P}\left\{\xi \mid \mathcal{F}_{m}\right\}, \mathcal{F}_{m}\right\}_{m=0}^{\infty}, P \in M,
$$

is a local regular super-martingale relative to the convex set of equivalent measures $M$.

Proof. Due to Theorem 3, the random process $\left\{E^{P}\left\{\xi \mid \mathcal{F}_{m}\right\}, \mathcal{F}_{m}\right\}_{m=0}^{\infty}$ is a martingale relative to the convex set of equivalent measures $M$. Therefore,

$$
\begin{aligned}
& f_{m-1} E^{P}\left\{\xi \mid \mathcal{F}_{m-1}\right\}-E^{P}\left\{f_{m} E^{P}\left\{\xi \mid \mathcal{F}_{m}\right\} \mid \mathcal{F}_{m-1}\right\} \\
& =E^{P}\left\{\left(f_{m-1}-f_{m}\right) E^{P}\left\{\xi \mid \mathcal{F}_{m}\right\} \mid \mathcal{F}_{m-1}\right\}, m=\overline{1, \infty} .
\end{aligned}
$$

So, if to put $\bar{g}_{m}^{0}=\left(f_{m-1}-f_{m}\right) E^{P}\left\{\xi \mid \mathcal{F}_{m}\right\}, m=\overline{1, \infty}$, then $\bar{g}_{m}^{0} \geq 0$, it is $\mathcal{F}_{m}$ measurable and $E^{P} \bar{g}_{m}^{0} \leq E^{P} \xi\left(\left|f_{m-1}\right|+\left|f_{m}\right|\right)<\infty$. It proves the needed statement.

Corollary 1. If $f_{m}=\alpha, m=\overline{1, \infty}, \alpha \in R^{1}, \xi \in A_{0}$, then $\left\{\alpha E^{P}\left\{\xi \mid \mathcal{F}_{m}\right\}, \mathcal{F}_{m}\right\}_{m=0}^{\infty}$ is a local regular martingale. Assume that $\xi=1$, then $\left\{f_{m}, \mathcal{F}_{m}\right\}_{m=0}^{\infty}$ is a local regular super-martingale relative to a convex set of equivalent measures $M$.

Denote $F_{0}$ the set of adapted processes

$$
F_{0}=\left\{f=\left\{f_{m}\right\}_{m=0}^{\infty}, P\left(\left|f_{m}\right|<\infty\right)=1, P \in M, f_{m} \leq f_{m-1}\right\} .
$$

For every $\xi \in A_{0}$ let us introduce the set of adapted processes

$$
L_{\xi}=\left\{\bar{f}=\left\{f_{m} E^{P}\left\{\xi \mid \mathcal{F}_{m}\right\}\right\}_{m=0}^{\infty},\left\{f_{m}\right\}_{m=0}^{\infty} \in F_{0}, E^{P} \xi\left|f_{m}\right|<\infty, P \in M\right\},
$$

and

$$
V=\bigcup_{\xi \in A_{0}} L_{\xi}
$$

Corollary 2. Every random process from the set $K$, where

$$
K=\left\{\sum_{i=1}^{m} C_{i} \bar{f}_{i}, \bar{f}_{i} \in V, C_{i} \geq 0, i=\overline{1, m}, m=\overline{1, \infty}\right\},
$$

is a local regular super-martingale relative to the convex set of equivalent measures $M$ on a measurable space $\{\Omega, \mathcal{F}\}$ with filtration $\mathcal{F}_{m}$ on it.

Proof. The proof is evident.

Theorem 5. On a measurable space $\{\Omega, \mathcal{F}\}$ and a filtration $\mathcal{F}_{n}$ on it, let $M$ be an arbitrary convex set of equivalent measures. Suppose that $\left\{f_{m}, \mathcal{F}_{m}\right\}_{m=0}^{\infty}$ is a nonnegative uniformly integrable super-martingale relative to a convex set of 
equivalent measures $M$, then the necessary and sufficient conditions for it to be a local regular one is belonging it to the set $K$.

Proof. Necessity. It is evident that if $\left\{f_{m}, \mathcal{F}_{m}\right\}_{m=0}^{\infty}$ belongs to $K$, then it is a local regular super-martingale.

Sufficiency. Suppose that $\left\{f_{m}, \mathcal{F}_{m}\right\}_{m=0}^{\infty}$ is a local regular super-martingale. Then there exists nonnegative adapted process $\left\{\overline{\boldsymbol{g}}_{m}^{0}\right\}_{m=1}^{\infty}, E^{P} \overline{\boldsymbol{g}}_{m}^{0}<\infty, m=\overline{1, \infty}$, and a martingale $\left\{M_{m}\right\}_{m=0}^{\infty}$, such that

$$
f_{m}=M_{m}-\sum_{i=1}^{m} \bar{g}_{i}^{0}, m=\overline{0, \infty} .
$$

Then $M_{m} \geq 0, m=\overline{0, \infty}, E^{P} M_{m}<\infty, P \in M$. Since $0<E^{P} M_{m}=f_{0}<\infty$ we have $E^{P} \sum_{i=1}^{m} \bar{g}_{i}^{0}<f_{0}$. Let us put $g_{\infty}=\lim _{m \rightarrow \infty} \sum_{i=1}^{m} \bar{g}_{i}^{0}$. Using the uniform integrability of $f_{m}$, we can pass to the limit in the equality

$$
E^{P}\left(f_{m}+\sum_{i=1}^{m} \bar{g}_{i}^{0}\right)=f_{0}, \quad P \in M,
$$

as $m \rightarrow \infty$. Passing to the limit in the last equality, as $m \rightarrow \infty$, we obtain

$$
E^{P}\left(f_{\infty}+g_{\infty}\right)=f_{0}, P \in M .
$$

Introduce into consideration a random value $\xi=\frac{f_{\infty}+g_{\infty}}{f_{0}}$. Then $E^{P} \xi=1, P \in M$. From here we obtain that $\xi \in A_{0}$ and

$$
M_{m}=f_{0} E^{P}\left\{\xi \mid \mathcal{F}_{m}\right\}, m=\overline{0, \infty} .
$$

Let us put $\bar{f}_{m}^{2}=-\sum_{i=1}^{m} \bar{g}_{i}^{0}$. It is easy to see that the adapted random process $\bar{f}_{2}=\left\{\bar{f}_{m}^{2}, \mathcal{F}_{m}\right\}_{m=0}^{\infty}$ belongs to $F_{0}$. Therefore, for the super-martingale $f=\left\{f_{m}, \mathcal{F}_{m}\right\}_{m=0}^{\infty}$ the representation

$$
f=\bar{f}_{1}+\bar{f}_{2}
$$

is valid, where $\bar{f}_{1}=\left\{f_{0} E^{P}\left\{\xi \mid \mathcal{F}_{m}\right\}, \mathcal{F}_{m}\right\}_{m=0}^{\infty}$ belongs to $L_{\xi}$ with $\xi=\frac{f_{\infty}+g_{\infty}}{f_{0}}$ and $f_{m}^{1}=f_{0}, m=\overline{0, \infty}$. The same is valid for $\bar{f}_{2}$ with $\xi=1$. This implies that $f$ belongs to the set $K$. Theorem 5 is proved.

Theorem 6. On a measurable space $\{\Omega, \mathcal{F}\}$ and a filtration $\mathcal{F}_{n}$ on it, let $M$ be an arbitrary convex set of equivalent measures. Suppose that the super-martingale $\left\{f_{m}, \mathcal{F}_{m}\right\}_{m=0}^{\infty}$ relative to the convex set of equivalent measures $M$ satisfy conditions

$$
\left|f_{m}\right| \leq C \xi_{0}, m=\overline{1, \infty}, \xi_{0} \in A_{0}, 0<C<\infty,
$$

then the necessary and sufficient conditions for it to be a local regular one is belonging it to the set $K$.

Proof. The necessity is evident.

Sufficiency. Suppose that $\left\{f_{m}, \mathcal{F}_{m}\right\}_{m=0}^{\infty}$ is a local regular super-martingale. 
Then there exists a nonnegative adapted random process $\left\{\bar{g}_{m}^{0}\right\}_{m=1}^{\infty}, E^{P} \bar{g}_{m}^{0}<\infty, m=\overline{1, \infty}$, and a martingale $\left\{M_{m}\right\}_{m=0}^{\infty}, E^{P}\left|M_{m}\right|<\infty, m=\overline{1, \infty}, P \in M$, such that

$$
f_{m}=M_{m}-\sum_{i=1}^{m} \bar{g}_{i}^{0}, m=\overline{0, \infty} .
$$

The inequalities $f_{m}+C \xi_{0} \geq 0, m=\overline{1, \infty}$, give the inequalities

$$
f_{m}+C E^{P}\left\{\xi_{0} \mid \mathcal{F}_{m}\right\} \geq 0, m=\overline{0, \infty} .
$$

From the inequalities (58) it follows that the super-martingale $\left\{f_{m}, \mathcal{F}_{m}\right\}_{m=0}^{\infty}$ is a uniformly integrable one relative to the convex set of equivalent measures $M$. The martingale $\left\{E^{P}\left\{\xi_{0} \mid \mathcal{F}_{m}\right\}, \mathcal{F}_{m}\right\}_{m=0}^{\infty}$ relative to the convex set of equivalent measures $M$ is also uniformly integrable one.

Then $M_{m}+C E^{P}\left\{\xi_{0} \mid \mathcal{F}_{m}\right\} \geq 0, m=\overline{0, \infty}$. Since $0<E^{P}\left[M_{m}+C E^{P}\left\{\xi_{0} \mid \mathcal{F}_{m}\right\}\right]=f_{0}+C<\infty$ we have $E^{P} \sum_{i=1}^{m} \bar{g}_{i}^{0}<f_{0}+C$. Let us put $g_{\infty}=\lim _{m \rightarrow \infty} \sum_{i=1}^{m} \bar{g}_{i}^{0}$. Using the uniform integrability of $f_{m}$ and $\sum_{i=1}^{m} \bar{g}_{i}^{0}$ we can pass to the limit in the equality

$$
E^{P}\left(f_{m}+\sum_{i=1}^{m} \bar{g}_{i}^{0}\right)=f_{0}, P \in M,
$$

as $m \rightarrow \infty$. Passing to the limit in the last equality, as $m \rightarrow \infty$, we obtain

$$
E^{P}\left(f_{\infty}+g_{\infty}\right)=f_{0}, P \in M .
$$

Introduce into consideration a random value $\xi_{1}=\frac{f_{\infty}+C \xi_{0}+g_{\infty}}{f_{0}+C} \geq 0$. Then $E^{P} \xi_{1}=1, P \in M$. From here we obtain that $\xi_{1} \in A_{0}$ and for the super-martingale $f=\left\{f_{m}, \mathcal{F}_{m}\right\}_{m=0}^{\infty}$ the representation

$$
f_{m}=f_{m}^{0} E^{P}\left\{\xi_{0} \mid \mathcal{F}_{m}\right\}+f_{m}^{1} E^{P}\left\{\xi_{1} \mid \mathcal{F}_{m}\right\}+f_{m}^{2} E^{P}\left\{\xi_{2} \mid \mathcal{F}_{m}\right\}, m=\overline{0, \infty},
$$

is valid, where $f_{m}^{0}=-C, f_{m}^{1}=f_{0}+C, f_{m}^{2}=-\sum_{i=1}^{m} \bar{g}_{i}^{0}, m=\overline{0, \infty}, \xi_{2}=1$. From the last representation it follows that the super-martingale $f=\left\{f_{m}, \mathcal{F}_{m}\right\}_{m=0}^{\infty}$ belongs to the set $K$. Theorem 6 is proved.

Corollary 3. Let $f_{N}, N<\infty$ be a $\mathcal{F}_{N}$-measurable integrable random value, $\sup _{P \in M} E^{P}\left|f_{N}\right|<\infty$, and let there exist $\alpha_{0} \in R^{1}$ such that

$$
-\alpha_{0} M_{N}+f_{N} \leq 0, \omega \in \Omega,
$$

where $\left\{M_{m}, \mathcal{F}_{m}\right\}_{m=0}^{\infty}=\left\{E^{P}\left\{\xi \mid \mathcal{F}_{m}\right\}, \mathcal{F}_{m}\right\}_{m=0}^{\infty}, \xi \in A_{0}$. Then a super-martingale $\left\{f_{m}^{0}+\bar{f}_{m}\right\}_{m=0}^{\infty}$ is a local regular one relative to the convex set of equivalent measures $M$, where

$$
f_{m}^{0}=\alpha_{0} M_{m},
$$




$$
\bar{f}_{m}= \begin{cases}0, & m<N, \\ f_{N}-\alpha_{0} M_{N}, & m \geq N .\end{cases}
$$

Proof. It is evident that $\bar{f}_{m-1}-\bar{f}_{m} \geq 0, m=\overline{0, \infty}$. Therefore, the super-martingale

$$
f_{m}^{0}+\bar{f}_{m}= \begin{cases}\alpha_{0} M_{m}, & m<N, \\ f_{N}, & m=N, \\ f_{N}-\alpha_{0} M_{N}+\alpha_{0} M_{m}, & m>N\end{cases}
$$

is a local regular one relative to the convex set of equivalent measures $M$. Corollary 3 is proved.

\section{Optional Decomposition for Super-Martingales Relative to the Complete Convex Set of Equivalent Measures}

In this section we introduce the notion of complete set of equivalent measures and prove that non negative super-martingales are local regular ones with respect to this set of measures. For this purpose we are needed the next auxiliary statement.

Theorem 7. The necessary and sufficient conditions of the local regularity of the nonnegative super-martingale $\left\{f_{m}, \mathcal{F}_{m}\right\}_{m=0}^{\infty}$ relative to a convex set of equivalent measures $M$ are the existence of $\mathcal{F}_{m}$-measurable random values $\xi_{m}^{0} \in A_{0}, m=\overline{1, \infty}$, such that

$$
\frac{f_{m}}{f_{m-1}} \leq \xi_{m}^{0}, E^{P}\left\{\xi_{m}^{0} \mid \mathcal{F}_{m-1}\right\}=1, \quad P \in M, m=\overline{1, \infty} .
$$

Proof. The necessity. Without loss of generality, we assume that $f_{m} \geq a$ for a certain real number $a>0$. Really, if it is not so, then we can come to the consideration of the super-martingale $\left\{f_{m}+a, \mathcal{F}_{m}\right\}_{m=0}^{\infty}$ Thus, let $\left\{f_{m}, \mathcal{F}_{m}\right\}_{m=0}^{\infty}$ be a nonnegative local regular super-martingale. Then there exists a nonnegative adapted random process $\left\{g_{m}\right\}_{m=0}^{\infty}, g_{0}=0$, such that $\sup _{P \in M} E^{P} g_{m}<\infty$,

$$
f_{m-1}-E^{P}\left\{f_{m} \mid \mathcal{F}_{m-1}\right\}=E^{P}\left\{g_{m} \mid \mathcal{F}_{m-1}\right\}, P \in M, m=\overline{1, \infty} .
$$

Let us put $\xi_{m}^{0}=\frac{f_{m}+g_{m}}{f_{m-1}}, m=\overline{1, \infty}$. Then $\xi_{m}^{0} \in A_{0}$ and from the equalities (68) we obtain $E^{P}\left\{\xi_{m}^{0} \mid \mathcal{F}_{m-1}\right\}=1, P \in M, m=\overline{1, \infty}$. It is evident that the inequalities (67) are valid.

The sufficiency. Suppose that the conditions of Theorem 7 are valid. Then $f_{m} \leq f_{m-1}+f_{m-1}\left(\xi_{m}^{0}-1\right)$. Introduce the denotation $g_{m}=-f_{m}+f_{m-1} \xi_{m}^{0}$. Then $g_{m} \geq 0, \sup _{P \in M} E^{P} g_{m} \leq \sup _{P \in M} E^{P} f_{m}+\sup _{P \in M} E^{P} f_{m-1}<\infty, m=\overline{1, \infty}$. The last equality and inequalities give

$$
f_{m}=f_{0}+\sum_{i=1}^{m} f_{i-1}\left(\xi_{i}^{0}-1\right)-\sum_{i=1}^{m} g_{i}, \quad m=\overline{1, \infty} .
$$

Let us consider the random process $\left\{M_{m}, \mathcal{F}_{m}\right\}_{m=0}^{\infty}$, where 
$M_{m}=f_{0}+\sum_{i=1}^{m} f_{i-1}\left(\xi_{i}^{0}-1\right)$. Then $E^{P}\left\{M_{m} \mid \mathcal{F}_{m-1}\right\}=M_{m-1}, P \in M, m=\overline{1, \infty}$. Theorem 7 is proved.

\subsection{Space of Finite Set of Elementary Events}

In this subsection we assume that a space of elementary events $\Omega$ is finite one, that is, $N_{0}=|\Omega|<\infty$, and we give a new proof of the optional decomposition for super-martingales relative to the complete convex set of equivalent measures. This proof does not use topological arguments as in [15].

Let $\mathcal{F}$ be a certain algebra of subsets of the set $\Omega$ and let $\mathcal{F}_{n} \subset \mathcal{F}_{n+1} \subset \mathcal{F}$ be an increasing set of algebras, where $\mathcal{F}_{0}=\{\varnothing, \Omega\}, \mathcal{F}_{N}=\mathcal{F}$. Denote $M$ a convex set of equivalent measures on a measurable space $\{\Omega, \mathcal{F}\}$. Further, we assume that the set $A_{0}$ contains an element $\xi_{0} \neq 1$. It is evident that every algebra $\mathcal{F}_{n}$ is generated by sets $A_{i}^{n}, i=\overline{1, N_{n}}, A_{i}^{n} \cap A_{j}^{n}=\varnothing, i \neq j, \quad N_{n}<\infty$,

$\bigcup_{i=1}^{N_{n}} A_{i}^{n}=\Omega, n=\overline{1, N}$. Let $m_{n}=E^{P}\left\{\xi_{0} \mid \mathcal{F}_{n}\right\}, P \in M, n=\overline{1, N}$. Then for $m_{n}$ the representation

$$
m_{n}=\sum_{i=1}^{N_{n}} m_{i}^{n} \chi_{A_{i}^{n}}(\omega), n=\overline{1, N}
$$

is valid. Consider the difference $d^{n}(\omega)=m_{n}-m_{n-1}$. Then

$$
\begin{gathered}
d^{n}(\omega)=\sum_{j=1}^{N_{n}} d_{j}^{n} \chi_{A_{j}^{n}}(\omega)=\sum_{j \in I_{n}^{-}} d_{j}^{n} \chi_{A_{j}^{n}}(\omega)+\sum_{j \in I_{n}^{+}} d_{j}^{n} \chi_{A_{j}^{n}}(\omega), \\
\sum_{j \in I_{n}^{-}} \chi_{A_{j}^{n}}(\omega)+\sum_{j \in I_{n}^{+}} \chi_{A_{j}^{n}}(\omega)=1,
\end{gathered}
$$

where $d_{j}^{n} \leq 0$, as $j \in I_{n}^{-}$, and $d_{j}^{n}>0$ for $j \in I_{n}^{+}$. From the equalities (71), (72) we obtain

$$
\begin{gathered}
E^{P} d^{n}(\omega)=\sum_{j \in I_{n}^{-}} d_{j}^{n} P\left(A_{j}^{n}\right)+\sum_{j \in I_{n}^{+}} d_{j}^{n} P\left(A_{j}^{n}\right)=0, P \in M, \\
\sum_{j \in I_{n}^{-}} P\left(A_{j}^{n}\right)+\sum_{j \in I_{n}^{+}} P\left(A_{j}^{n}\right)=1, P \in M .
\end{gathered}
$$

Denote $M_{n}$ the contraction of the set of measures $M$ on the algebra $\mathcal{F}_{n}$. Introduce into the set $M_{n}$ the metrics

$$
\rho_{n}\left(P_{1}, P_{2}\right)=\max _{B} \sum_{s=1}^{k}\left|P_{1}\left(B_{s}^{n}\right)-P_{2}\left(B_{s}^{n}\right)\right|, P_{1}, P_{2} \in M_{n}, n=\overline{1, N},
$$

where $B=\left\{B_{1}^{n}, \cdots, B_{k}^{n}\right\}$ is a partition of $\Omega$ on $k$ subsets, that is, $B_{i}^{n} \in F_{n}, i=\overline{1, k}$, $B_{i}^{n} \cap B_{j}^{n}=\varnothing, i \neq j, \bigcup_{i=1}^{k} B_{i}^{n}=\Omega$. The maximum in the formula (75) is all over the partitions of the set $\Omega$, belonging to the $\sigma$-algebra $\mathcal{F}_{n}$.

Definition 3. On a measurable space $\{\Omega, \mathcal{F}\}$, a convex set of equivalent measure $M$ we call complete if for every $1 \leq n \leq N$ the closure of the set of measures $M_{n}$ in the metrics (75) contains the measures 


$$
P_{i j}^{n}(A)= \begin{cases}0, & A \neq A_{i}^{n}, A_{j}^{n}, \\ \frac{d_{j}^{n}}{-d_{i}^{n}+d_{j}^{n}}, & A=A_{i}^{n}, \\ \frac{-d_{i}^{n}}{-d_{i}^{n}+d_{j}^{n}}, & A=A_{j}^{n}\end{cases}
$$

for every $i \in I_{n}^{-}$and $j \in I_{n}^{+}$.

Lemma 10. Let a convex family of equivalent measures $M$ be a complete one and the set $A_{0}$ contains an element $\xi_{0} \neq 1$. Then for every non negative $\mathcal{F}_{n}$ measurable random value $\xi_{n}=\sum_{i=1}^{N_{n}} C_{i}^{n} \chi_{A_{i}^{n}}$ there exists a real number $\alpha_{n}$ such that

$$
\frac{\sum_{i=1}^{N_{n}} C_{i}^{n} \chi_{A_{i}^{n}}}{\sup _{P \in M_{n}} \sum_{i=1}^{N_{n}} C_{i}^{n} P\left(A_{i}^{n}\right)} \leq 1+\alpha_{n}\left(m_{n}-m_{n-1}\right), n=\overline{1, N} .
$$

Proof. On the set $\bar{M}_{n}$, the functional $\varphi(P)=\sum_{i=1}^{N_{n}} C_{i}^{n} P\left(A_{i}^{n}\right)$ is a continuous one, where $\bar{M}_{n}$ is the closure of the set $M_{n}$ in the metrics $\rho_{n}\left(P_{1}, P_{2}\right)$. From this it follows that the equality

$$
\sup _{P \in M_{n}} \sum_{i=1}^{N_{n}} C_{i}^{n} P\left(A_{i}^{n}\right)=\sup _{P \in \bar{M}_{n}} \sum_{i=1}^{N_{n}} C_{i}^{n} P\left(A_{i}^{n}\right)
$$

is valid. Denote

$$
\begin{gathered}
f_{i}^{n}=\frac{C_{i}^{n}}{\sup _{P \in M_{n}} \sum_{i=1}^{N_{n}} C_{i}^{n} P\left(A_{i}^{n}\right)}, i=\overline{1, N_{n}} \text {. Then } \\
\sum_{i=1}^{N_{n}} f_{i}^{n} P\left(A_{i}^{n}\right) \leq 1, P \in \bar{M}_{n} .
\end{gathered}
$$

For those $i \in I_{n}^{-}$for which $d_{i}^{n}<0$ and those $j \in I_{n}^{+}$for which $d_{j}^{n}>0$ the inequality (79) is as follows

$$
f_{i}^{n} \frac{d_{j}^{n}}{-d_{i}^{n}+d_{j}^{n}}+\frac{-d_{i}^{n}}{-d_{i}^{n}+d_{j}^{n}} f_{j}^{n} \leq 1, d_{i}^{n}<0, i \in I^{-}, d_{j}^{n}>0, j \in I_{n}^{+} .
$$

From (80) we obtain the inequalities

$$
f_{j}^{n} \leq 1+\frac{1-f_{i}^{n}}{-d_{i}^{n}} d_{j}^{n}, d_{i}^{n}<0, i \in I_{n}^{-}, d_{j}^{n}>0, j \in I_{n}^{+} .
$$

Since the inequalities (81) are valid for every $\frac{1-f_{i}^{n}}{-d_{i}^{n}}$, as $d_{i}^{n}<0$, and since the set of such elements is finite, then if to denote

$$
\alpha_{n}=\min _{\left\{i, d_{i}^{n}<0\right\}} \frac{1-f_{i}^{n}}{-d_{i}^{n}},
$$

then we have

$$
f_{j}^{n} \leq 1+\alpha_{n} d_{j}^{n}, d_{j}^{n}>0, \quad j \in I_{n}^{+} .
$$


From the definition of $\alpha_{n}$ we obtain the inequalities

$$
f_{i}^{n} \leq 1+\alpha_{n} d_{i}^{n}, d_{i}^{n}<0, \quad i \in I_{n}^{-} .
$$

Now if $d_{i}^{n}=0$ for some $i \in I_{n}^{-}$, then in this case $f_{i}^{n} \leq 1$. All these inequalities give

$$
f_{i}^{n} \leq 1+\alpha_{n} d_{i}^{n}, i \in I_{n}^{-} \cup I_{n}^{+} .
$$

Multiplying on $\chi_{A_{i}^{n}}$ the inequalities (85) and summing over all $i \in I_{n}^{-} \cup I_{n}^{+}$ we obtain the needed inequality. Lemma 10 is proved.

Theorem 8. Suppose that the conditions of Lemma 10 are valid. Then every non negative super-martingale $\left\{f_{m}, \mathcal{F}_{m}\right\}_{m=0}^{N}$ relative to a convex set of equivalent measures $M$, satisfying conditions

$$
\frac{f_{n}}{f_{n-1}} \leq C_{n}<\infty, n=\overline{1, N}
$$

is a local regular one, where $C_{n}, n=\overline{1, N}$ are constants.

Proof. Consider the random value $\xi_{n}=\frac{f_{n}}{f_{n-1}}$. Due to Lemma 10

$$
\frac{\xi_{n}}{\sup _{P \in M} E^{P} \xi_{n}} \leq 1+\alpha_{n}\left(m_{n}-m_{n-1}\right)=\xi_{n}^{0}, \quad n=\overline{1, N} \text {. }
$$

It is evident that $E^{P}\left\{\xi_{n}^{0} \mid \mathcal{F}_{n-1}\right\}=1, P \in M, n=\overline{1, N}$. Since $\sup _{P \in M} E^{P} \xi_{n} \leq 1$, then

$$
\frac{f_{n}}{f_{n-1}} \leq \xi_{n}^{0}, n=\overline{1, N} \text {. }
$$

Theorem 7 and the inequalities (88) prove Theorem 8.

Theorem 9. On a finite space of elementary events $\{\Omega, \mathcal{F}\}$ with a filtration $\mathcal{F}_{n}$ on it, every super-martingale $\left\{f_{m}, \mathcal{F}_{m}\right\}_{m=0}^{N}$ relative to the complete convex set of equivalent measures $M$ is a local regular one if the set $A_{0}$ contains $\xi_{0} \neq 1$.

Proof. It is evident that every super-martingale $\left\{f_{m}, \mathcal{F}_{m}\right\}_{m=0}^{N}$ is bounded. Therefore, there exists a constant $C_{0}>0$ such that $\frac{3 C_{0}}{2}>f_{m}+C_{0}>\frac{C_{0}}{2}, \omega \in \Omega, m=\overline{0, N}$. From this it follows that the super-martingale $\left\{f_{m}+C_{0}, \mathcal{F}_{m}\right\}_{m=0}^{N}$ is a nonnegative one and satisfies the conditions

$$
\frac{f_{n}+C_{0}}{f_{n-1}+C_{0}} \leq 3, n=\overline{1, N}
$$

It implies that the conditions of Theorem 8 are satisfied. Theorem 9 is proved.

Theorem 10. Let $M$ be a complete convex set of equivalent measure on a measurable space $\{\Omega, \mathcal{F}\}$ with a filtration $\mathcal{F}_{m}$ on it. Suppose that $\xi_{0} \in A_{0}, \xi_{0} \neq 1$, and $m_{n}=E^{P}\left\{\xi_{0} \mid \mathcal{F}_{n}\right\}$ is a martingale relative to the set of measures $M$. Let $M_{0}^{a}$ be a set of all martingale measures absolutely continuous 
relative to any measure $P \in M$. Then the inclusion $\bar{M} \subseteq M_{0}^{a}$ is valid, where $\bar{M}$ is a closure of the set of measures $M$ in metrics $\rho_{N}\left(P_{1}, P_{2}\right)$, defined in (75).

Proof. Let the sequence $P_{s} \in M$ be a convergent one to the measure $P_{0} \in \bar{M}$, then for $D \in \mathcal{F}_{n-1}$

$$
\int_{D} m_{n} \mathrm{~d} P_{s}=\int_{D} m_{n-1} \mathrm{~d} P_{s}, s=\overline{1, \infty} .
$$

The functionals $\int m_{n} \mathrm{~d} P, \int m_{n-1} \mathrm{~d} P$ on the set $\bar{M}$ for all $D \in \mathcal{F}_{n-1}$ are continuous ones relative ${ }^{D}$ to the ${ }^{D}$ metrics $\rho_{N}\left(P_{1}, P_{2}\right)$, defined by the formula (75). Going to the limit in the equality (90), as $s \rightarrow \infty$, we obtain

$$
\int_{D} m_{n} \mathrm{~d} P_{0}=\int_{D} m_{n-1} \mathrm{~d} P_{0}, n=\overline{1, N}, \quad D \in \mathcal{F}_{n-1} .
$$

The last implies that $P_{0} \in M_{0}^{a}$. Theorem 10 is proved.

\subsection{Countable Set of Elementary Events}

In this subsection, we generalize the results of the previous subsection onto the countable space of elementary events. Let $\mathcal{F}$ be a certain $\sigma$-algebra of subsets of the countable set of elementary events $\Omega$ and let $\mathcal{F}_{n} \subset \mathcal{F}_{n+1} \subset \mathcal{F}$ be a certain increasing set of $\sigma$-algebras, where $\mathcal{F}_{0}=\{\varnothing, \Omega\}$. Denote $M$ a set of equivalent measures on the measurable space $\{\Omega, \mathcal{F}\}$. Further, we assume that the set $A_{0}$ contains an element $\xi_{0} \neq 1$. Suppose that the $\sigma$-algebra $\mathcal{F}_{n}$ is generated by the sets $A_{i}^{n}, i=\overline{1, \infty}, A_{i}^{n} \cap A_{j}^{n}=\varnothing, i \neq j, \bigcup_{i=1}^{\infty} A_{i}^{n}=\Omega, n=\overline{1, \infty}$.

Introduce into consideration the martingale $m_{n}=E^{P}\left\{\xi_{0} \mid \mathcal{F}_{n}\right\}, P \in M, n=\overline{1, \infty}$. Then for $m_{n}$ the representation

$$
m_{n}=\sum_{i=1}^{\infty} m_{i}^{n} \chi_{A_{i}^{n}}(\omega), n=\overline{1, \infty},
$$

is valid. Consider the difference $d^{n}(\omega)=m_{n}-m_{n-1}$. Then

$$
\begin{gathered}
d^{n}(\omega)=\sum_{j=1}^{\infty} d_{j}^{n} \chi_{A_{j}^{n}}(\omega)=\sum_{j \in I^{-}} d_{j}^{n} \chi_{A_{j}^{n}}(\omega)+\sum_{j \in I^{+}} d_{j}^{n} \chi_{A_{j}^{n}}(\omega), \\
\sum_{j \in I^{-}} \chi_{A_{j}^{n}}(\omega)+\sum_{j \in I^{+}} \chi_{A_{j}^{n}}(\omega)=1,
\end{gathered}
$$

where $d_{j}^{n} \leq 0$, as $j \in I_{n}^{-}$, and $d_{j}^{n}>0, j \in I_{n}^{+}$. From the equalities (93), (94) we obtain

$$
\begin{gathered}
E^{P} d^{n}(\omega)=\sum_{j \in I_{n}^{-}} d_{j}^{n} P\left(A_{j}^{n}\right)+\sum_{j \in I_{n}^{+}} d_{j}^{n} P\left(A_{j}^{n}\right)=0, \quad P \in M, \\
\sum_{j \in I_{n}^{-}} P\left(A_{j}^{n}\right)+\sum_{j \in I_{n}^{+}} P\left(A_{j}^{n}\right)=1, \quad P \in M .
\end{gathered}
$$

Denote $M_{n}$ the contraction of the set of measures $M$ on the $\sigma$-algebra $\mathcal{F}_{n}$. Introduce into the set $M_{n}$ the metrics

$$
\rho_{n}\left(P_{1}, P_{2}\right)=\sup _{B} \sum_{s=1}^{k}\left|P_{1}\left(B_{s}^{n}\right)-P_{2}\left(B_{s}^{n}\right)\right|, P_{1}, P_{2} \in M_{n}, n=\overline{1, \infty},
$$


where $B=\left\{B_{1}^{n}, \cdots, B_{k}^{n}\right\}$ is a partition of $\Omega$ on $k$ subsets, that is, $B_{i}^{n} \in \mathcal{F}_{n}, i=\overline{1, k}$, $B_{i}^{n} \cap B_{j}^{n}=\varnothing, i \neq j, \bigcup_{i=1}^{k} B_{i}^{n}=\Omega$. The supremum in the formula (97) is all over the partitions of the set $\Omega$, belonging to the $\sigma$-algebra $\mathcal{F}_{n}$.

Definition 4. On a measurable space $\{\Omega, \mathcal{F}\}$ with a filtration $\mathcal{F}_{n}$ on it, a convex set of equivalent measure $M$ we call complete one if for every $1 \leq n<\infty$ the closure of the set of measures $M_{n}$ in the metrics (97) contains the measures

$$
P_{i j}^{n}(A)= \begin{cases}0, & A \neq A_{i}^{n}, A_{j}^{n}, \\ \frac{d_{j}^{n}}{-d_{i}^{n}+d_{j}^{n}}, & A=A_{i}^{n}, \\ \frac{-d_{i}^{n}}{-d_{i}^{n}+d_{j}^{n}}, & A=A_{j}^{n}\end{cases}
$$

for every $i \in I_{n}^{-}$and $j \in I_{n}^{+}$.

Lemma 11. Let a family of measures $M$ be complete and the set $A_{0}$ contains an element $\xi_{0} \neq 1$. Then for every non-negative bounded $\mathcal{F}_{n}$-measurable random value $\xi_{n}=\sum_{i=1}^{\infty} C_{i}^{n} \chi_{A_{i}^{n}}$ there exists a real number $\alpha_{n}$ such that

$$
\frac{\sum_{i=1}^{\infty} C_{i}^{n} \chi_{A_{i}^{n}}}{\sup _{P \in M_{n}} \sum_{i=1}^{\infty} C_{i}^{n} P\left(A_{i}^{n}\right)} \leq 1+\alpha_{n}\left(m_{n}-m_{n-1}\right), n=\overline{1, \infty .}
$$

Proof. On the set $\bar{M}_{n}$, the functional $\varphi(P)=\sum_{i=1}^{\infty} C_{i}^{n} P\left(A_{i}^{n}\right)$ is a continuous one relative to the metrics $\rho_{n}\left(P_{1}, P_{2}\right)$, where $\bar{M}_{n}$ is the closure of the set $M_{n}$ in this metrics. From this it follows that the equality

$$
\sup _{P \in M_{n}} \sum_{i=1}^{\infty} C_{i}^{n} P\left(A_{i}^{n}\right)=\sup _{P \in M_{n}} \sum_{i=1}^{\infty} C_{i}^{n} P\left(A_{i}^{n}\right)
$$

is valid. Denote $f_{i}^{n}=\frac{C_{i}^{n}}{\sup _{P \in M_{n}} \sum_{i=1}^{\infty} C_{i}^{n} P\left(A_{i}^{n}\right)}, i=\overline{1, \infty}$. Then

$$
\sum_{i=1}^{\infty} f_{i}^{n} P\left(A_{i}^{n}\right) \leq 1, P \in \bar{M}_{n} .
$$

The last inequalities can be written in the form

$$
\sum_{i \in I^{-}} f_{i}^{n} P\left(A_{i}^{n}\right)+\sum_{i \in I^{+}} f_{i}^{n} P\left(A_{i}^{n}\right) \leq 1, P \in \bar{M}_{n} .
$$

For those $i \in I_{n}^{-}$for which $d_{i}^{n}<0$ and those $j \in I_{n}^{+}$for which $d_{j}^{n}>0$ the inequality (102) is as follows

$$
f_{i}^{n} \frac{d_{j}^{n}}{-d_{i}^{n}+d_{j}^{n}}+\frac{-d_{i}^{n}}{-d_{i}^{n}+d_{j}^{n}} f_{j}^{n} \leq 1, d_{i}^{n}<0, d_{j}^{n}>0, i \in I_{n}^{-}, j \in I_{n}^{+} .
$$

From (103) we obtain the inequalities 


$$
f_{j}^{n} \leq 1+\frac{1-f_{i}^{n}}{-d_{i}^{n}} d_{j}^{n}, d_{i}^{n}<0, d_{j}^{n}>0, i \in I_{n}^{-}, j \in I_{n}^{+} .
$$

Two cases are possible: 1 ) for all $\left.i \in I_{n}^{-}, \quad f_{i}^{n} \leq 1 ; 2\right)$ there exists $i \in I_{n}^{-}$such that $f_{i}^{n}>1$. First, let us consider the case a).

Since the inequalities (104) are valid for every $\frac{1-f_{i}^{n}}{-d_{i}^{n}}$, as $d_{i}^{n}<0$, and $f_{i}^{n} \leq 1, i \in I_{n}^{-}$, then if to denote

$$
\alpha_{n}=\inf _{\left\{i, d_{i}^{n}<0\right\}} \frac{1-f_{i}^{n}}{-d_{i}^{n}}
$$

we have $0 \leq \alpha_{n}<\infty$ and

$$
f_{j}^{n} \leq 1+\alpha_{n} d_{j}^{n}, d_{j}^{n}>0, \quad j \in I_{n}^{+} .
$$

From the definition of $\alpha_{n}$ we obtain the inequalities

$$
f_{i}^{n} \leq 1+\alpha_{n} d_{i}^{n}, d_{i}^{n}<0, \quad i \in I_{n}^{-} .
$$

Now, if $d_{i}^{n}=0$ for some $i \in I_{n}^{-}$, then in this case $f_{i}^{n} \leq 1$. All these inequalities give

$$
f_{i}^{n} \leq 1+\alpha_{n} d_{i}^{n}, i \in I_{n}^{-} \cup I_{n}^{+} .
$$

Consider the case b). From the inequality (104), we obtain

$$
f_{j}^{n} \leq 1-\frac{1-f_{i}^{n}}{d_{i}^{n}} d_{j}^{n}, d_{i}^{n}<0, d_{j}^{n}>0, i \in I_{n}^{-}, j \in I_{n}^{+} .
$$

The last inequalities give

$$
\frac{1-f_{i}^{n}}{d_{i}^{n}} \leq \min _{\left\{j, d_{j}^{n}>0\right\}} \frac{1}{d_{j}^{n}}<\infty, d_{i}^{n}<0, i \in I_{n}^{-} .
$$

Let us define $\alpha_{n}=\sup _{\left\{i, d_{i}^{n}<0\right\}} \frac{1-f_{i}^{n}}{d_{i}^{n}}<\infty$. Then from (109) we obtain

$$
f_{j}^{n} \leq 1-\alpha_{n} d_{j}^{n}, d_{j}^{n}>0, \quad j \in I_{n}^{+} .
$$

From the definition of $\alpha_{n}$, we have

$$
f_{i}^{n} \leq 1-\alpha_{n} d_{i}^{n}, d_{i}^{n}<0, \quad i \in I_{n}^{-} .
$$

The inequalities (111), (112) give

$$
f_{j}^{n} \leq 1-\alpha_{n} d_{j}^{n}, j \in I_{n}^{-} \cup I_{n}^{+} .
$$

Multiplying on $\chi_{A_{i}^{n}}$ the inequalities (108) and the inequalities (113) on $\chi_{A_{j}^{n}}$ and summing over all $i, j \in I_{n}^{-} \cup I_{n}^{+}$we obtain the needed inequality. The Lemma 11 is proved.

Theorem 11. Suppose that the conditions of Lemma 11 are valid. Then every non negative super-martingale $\left\{f_{m}, \mathcal{F}_{m}\right\}_{m=0}^{\infty}$ relative to a convex set of equivalent measures $M$, satisfying the conditions

$$
\frac{f_{m}}{f_{m-1}} \leq C_{m}<\infty, m=\overline{1, \infty}
$$


is a local regular one, where $C_{m}$ are constants.

Proof. From the conditions (114) it follows that $\sup _{P \in M} E^{P} f_{m}<\infty$ Consider the random value $\xi_{n}=\frac{f_{n}}{f_{n-1}}$. Due to Lemma 11

$$
\frac{\xi_{n}}{\sup _{P \in M} E^{P} \xi_{n}} \leq 1+\alpha_{n}\left(m_{n}-m_{n-1}\right)=\xi_{n}^{0} .
$$

It is evident that $E^{P}\left\{\xi_{n}^{0} \mid \mathcal{F}_{n-1}\right\}=1, P \in M, n=\overline{1, \infty}$. Since $\sup _{P \in M} E^{P} \xi_{n} \leq 1$, then

$$
\frac{f_{n}}{f_{n-1}} \leq \xi_{n}^{0}, n=\overline{1, \infty}
$$

Theorem 7 and the inequalities (116) prove Theorem 11.

\subsection{An arbitrary Space of Elementary Events}

In this subsection, we consider an arbitrary space of elementary events and prove the optional decomposition for non negative super-martingales.

Let $\mathcal{F}$ be a certain $\sigma$-algebra of subsets of the set of elementary events $\Omega$ and let $\mathcal{F}_{n} \subset \mathcal{F}_{n+1} \subset \mathcal{F}$ be an increasing set of the $\sigma$-algebras, where $\mathcal{F}_{0}=\{\varnothing, \Omega\}$. Denote $M$ a set of equivalent measures on a measurable space $\{\Omega, \mathcal{F}\}$ We assume that the $\sigma$-algebras $\mathcal{F}_{n}, n=\overline{1, \infty}$, and $\mathcal{F}$ are complete relative to any measure $P \in M$. Further, we suppose that the set $A_{0}$ contains an element $\xi_{0} \neq 1$. Let $m_{n}=E^{P}\left\{\xi_{0} \mid \mathcal{F}_{n}\right\}, P \in M, n=\overline{1, \infty}$.

Consider the difference $d^{n}(\omega)=m_{n}-m_{n-1}$. We assume that every $\omega \in \Omega$ belongs to the $\sigma$-algebra $\mathcal{F}_{n}, n=\overline{1, \infty}$, and $P(\{\omega\})=0, \omega \in \Omega, P \in M$.

For the random value $d^{n}(\omega)$ there exists not more then a countable set of the real number $d_{s}^{n}$ such that $P\left(A_{s}^{n}\right)>0$, where $A_{s}^{n}=\left\{\omega \in \Omega, d^{n}(\omega)=d_{s}^{n}\right\}$. It is evident that $A_{i}^{n} \cap A_{j}^{n}=\varnothing, i \neq j$. Suppose that $P\left(\Omega \backslash \bigcup_{i=1}^{\infty} A_{i}^{n}\right)>0$. Introduce for every $n$ two subsets $I_{n}^{-}=\left\{\omega \in \Omega, d^{n}(\omega) \leq 0\right\}, I_{n}^{+}=\left\{\omega \in \Omega, d^{n}(\omega)>0\right\}$ of the set $\left\{\omega \in \Omega,\left|d_{n}(\omega)\right|<\infty\right\}$.

Denote $M_{n}$ the contraction of the set of measures $M$ on the $\sigma$-algebra $\mathcal{F}_{n}$. Introduce into the set $M_{n}$ the metrics

$$
\rho_{n}\left(P_{1}, P_{2}\right)=\sup _{B} \sum_{s=1}^{k}\left|P_{1}\left(B_{s}^{n}\right)-P_{2}\left(B_{s}^{n}\right)\right|, P_{1}, P_{2} \in M_{n}, n=\overline{1, \infty}
$$

where $B=\left\{B_{1}^{n}, \cdots, B_{k}^{n}\right\}$ is a partition of $\Omega$ on $k$ subsets, that is, $B_{i}^{n} \in \mathcal{F}_{n}, i=\overline{1, k}$, $B_{i}^{n} \cap B_{j}^{n}=\varnothing, i \neq j, \bigcup_{i=1}^{k} B_{i}^{n}=\Omega$. The supremum in the formula (117) is all over the partitions of the set $\Omega$, belonging to the $\sigma$-algebra $\mathcal{F}_{n}$.

Definition 5. On a measurable space $\{\Omega, \mathcal{F}\}$ with filtration $\mathcal{F}_{n}$ on it, a convex set of equivalent measure $M$ we call complete if for every $1 \leq n<\infty$ the 
closure in metrics (117) of the set of measures $M_{n}$ contains the measures

$$
P_{\omega_{1}, \omega_{2}}^{n}(A)= \begin{cases}0, & \omega_{1}, \omega_{2} \in \Omega \backslash A, \\ \frac{d^{n}\left(\omega_{2}\right)}{-d^{n}\left(\omega_{1}\right)+d^{n}\left(\omega_{2}\right)}, & \omega_{1} \in A, A \cap\left\{\omega_{2}\right\}=\varnothing, \\ \frac{-d^{n}\left(\omega_{1}\right)}{-d^{n}\left(\omega_{1}\right)+d^{n}\left(\omega_{2}\right)}, & \omega_{2} \in \Omega \backslash A,(\Omega \backslash A) \cap\left\{\omega_{1}\right\}=\varnothing\end{cases}
$$

for $\omega_{1} \in I_{n}^{-}$and $\omega_{2} \in I_{n}^{+}$.

Lemma 12. Let a convex family of equivalent measures $M$ be a complete one and the set $A_{0}$ contains an element $\xi_{0} \neq 1$. Then for every non negative bounded $\mathcal{F}_{n}$-measurable random value $\xi_{n}$ there exists a real number $\alpha_{n}$ such that

$$
\frac{\xi_{n}}{\sup _{P \in M} E^{P} \xi_{n}} \leq 1+\alpha_{n}\left(m_{n}-m_{n-1}\right), n=\overline{1, \infty}
$$

Proof. On the set $\bar{M}_{n}$, the functional $\varphi(P)=\int \xi_{n} \mathrm{~d} P$ is a continuous one relative to the metrics $\rho_{n}\left(P_{1}, P_{2}\right)$, where $\bar{M}_{n}$ is thê closure of the set $M_{n}$ in this metrics. From this it follows that the equality

$$
\sup _{P \in M_{n}} \int_{\Omega} \xi_{n} \mathrm{~d} P=\sup _{P \in \bar{M}_{n}} \int_{\Omega} \xi_{n} \mathrm{~d} P
$$

is valid. Denote $f^{n}(\omega)=\frac{\xi_{n}(\omega)}{\sup _{P \in M_{n}} E^{P} \xi_{n}(\omega)}$. Then

$$
E^{P} f^{n}(\omega) \leq 1, P \in \bar{M}_{n} .
$$

The last inequalities can be written in the form

$$
\int_{I^{-}} f_{n}(\omega) \mathrm{d} P+\int_{I^{+}} f_{n}(\omega) \mathrm{d} P \leq 1, P \in \bar{M}_{n} .
$$

The inequality (122) for the measures (118) is as follows

$$
f^{n}\left(\omega_{1}\right) \frac{d^{n}\left(\omega_{2}\right)}{-d^{n}\left(\omega_{1}\right)+d^{n}\left(\omega_{2}\right)}+\frac{-d^{n}\left(\omega_{1}\right)}{-d^{n}\left(\omega_{1}\right)+d^{n}\left(\omega_{2}\right)} f^{n}\left(\omega_{2}\right) \leq 1, \omega_{1} \in I_{n}^{-}, \omega_{2} \in I_{n}^{+} .
$$

From (123) we obtain the inequalities

$$
\begin{gathered}
f^{n}\left(\omega_{2}\right) \leq 1+\frac{1-f^{n}\left(\omega_{1}\right)}{-d^{n}\left(\omega_{1}\right)} d^{n}\left(\omega_{2}\right), \\
d^{n}\left(\omega_{1}\right)<0, d^{n}\left(\omega_{2}\right)>0, \omega_{1} \in I_{n}^{-}, \omega_{2} \in I_{n}^{+} .
\end{gathered}
$$

Two cases are possible: 1) for all $\left.\omega_{1} \in I_{n}^{-}, f^{n}\left(\omega_{1}\right) \leq 1 ; 2\right)$ there exists $\omega_{1} \in I_{n}^{-}$ such that $f^{n}\left(\omega_{1}\right)>1$. First, let us consider the case a).

Since the inequalities (124) are valid for every $\frac{1-f^{n}\left(\omega_{1}\right)}{-d^{n}\left(\omega_{1}\right)}$, as $d^{n}\left(\omega_{1}\right)<0$, and $f^{n}\left(\omega_{1}\right) \leq 1, \omega_{1} \in I_{n}^{-}$, then if to denote 


$$
\alpha_{n}=\inf _{\left\{\omega_{1}, d^{n}\left(\omega_{1}\right)<0\right\}} \frac{1-f^{n}\left(\omega_{1}\right)}{-d^{n}\left(\omega_{1}\right)},
$$

we have $0 \leq \alpha_{n}<\infty$ and

$$
f^{n}\left(\omega_{2}\right) \leq 1+\alpha_{n} d^{n}\left(\omega_{2}\right), d^{n}\left(\omega_{2}\right)>0, \omega_{2} \in I_{n}^{+} .
$$

From the definition of $\alpha_{n}$ we obtain the inequalities

$$
f^{n}\left(\omega_{1}\right) \leq 1+\alpha_{n} d^{n}\left(\omega_{1}\right), d^{n}\left(\omega_{1}\right)<0, \omega_{1} \in I_{n}^{-} .
$$

Now, if $d^{n}\left(\omega_{1}\right)=0$ for some $\omega_{1} \in I_{n}^{-}$, then in this case $f^{n}\left(\omega_{1}\right) \leq 1$. All these inequalities give

$$
f^{n}(\omega) \leq 1+\alpha_{n} d^{n}(\omega), \omega \in I_{n}^{-} \cup I_{n}^{+} .
$$

Consider the case b). From the inequality (124), we obtain

$$
\begin{gathered}
f^{n}\left(\omega_{2}\right) \leq 1-\frac{1-f^{n}\left(\omega_{1}\right)}{d^{n}\left(\omega_{1}\right)} d^{n}\left(\omega_{2}\right), \\
d^{n}\left(\omega_{1}\right)<0, d^{n}\left(\omega_{2}\right)>0, \omega_{1} \in I_{n}^{-}, \omega_{2} \in I_{n}^{+} .
\end{gathered}
$$

The last inequalities give

$$
\frac{1-f^{n}\left(\omega_{1}\right)}{d^{n}\left(\omega_{1}\right)} \leq \inf _{\left\{\omega_{2}, d^{n}\left(\omega_{2}\right)>0\right\}} \frac{1}{d^{n}\left(\omega_{2}\right)}<\infty, d^{n}\left(\omega_{1}\right)<0, \omega_{1} \in I_{n}^{-} .
$$

Let us define $\alpha_{n}=\sup _{\left\{\omega_{1}, d^{n}\left(\omega_{1}\right)<0\right\}} \frac{1-f^{n}\left(\omega_{1}\right)}{d^{n}\left(\omega_{1}\right)}<\infty$. Then from (130) we obtain

$$
f^{n}\left(\omega_{2}\right) \leq 1-\alpha_{n} d^{n}\left(\omega_{2}\right), d^{n}\left(\omega_{2}\right)>0, \omega_{2} \in I_{n}^{+} .
$$

From the definition of $\alpha_{n}$ we have

$$
f^{n}\left(\omega_{1}\right) \leq 1-\alpha_{n} d^{n}\left(\omega_{1}\right), d^{n}\left(\omega_{1}\right)<0, \omega_{1} \in I_{n}^{-} .
$$

The inequalities (133), (134) give

$$
f^{n}(\omega) \leq 1-\alpha_{n} d^{n}(\omega), \omega \in I_{n}^{-} \cup I_{n}^{+} .
$$

Since the set $I_{n}^{-} U I_{n}^{+}$has probability one, Lemma 12 is proved.

Theorem 12. Suppose a convex set of equivalent measures $M$ is a complete one and the conditions of Lemma 12 are valid. Then every non negative super-martingale $\left\{f_{m}, \mathcal{F}_{m}\right\}_{m=0}^{\infty}$ relative to a convex set of equivalent measures $M$, satisfying conditions

$$
\frac{f_{m}}{f_{m-1}} \leq C_{m}<\infty, m=\overline{1, \infty}
$$

is a local regular one, where $C_{m}, m=\overline{1, \infty}$ are constants.

Proof. From the inequalities (136) it follows that $\sup _{P \in M} E^{P} f_{m}<\infty, m=\overline{1, \infty}$. Consider the random value $\xi_{n}=\frac{f_{n}}{f_{n-1}}$. Due to Lemma 12 


$$
\frac{\xi_{n}}{\sup _{P \in M} E^{P} \xi_{n}} \leq 1+\alpha_{n}\left(m_{n}-m_{n-1}\right)=\xi_{n}^{0} .
$$

It is evident that $E^{P}\left\{\xi_{n}^{0} \mid \mathcal{F}_{n-1}\right\}=1, P \in M, n=\overline{1, \infty}$. Since $\sup _{P \in M} E^{P} \xi_{n} \leq 1$, then

$$
\frac{f_{n}}{f_{n-1}} \leq \xi_{n}^{0}, n=\overline{1, \infty}
$$

Theorem 7 and the inequalities (138) prove Theorem 12.

Consequence 1 . If a super-martingale $\left\{f_{m}, \mathcal{F}_{m}\right\}_{m=0}^{\infty}$ relative to a complete convex set of equivalent measures $M$ satisfy conditions $0 \leq f_{m} \leq D_{m}, m=\overline{1, \infty}$, where $D_{m}<\infty$ are constant, then it is local regular.

Proof. The super-martingale $\left\{f_{m}+\varepsilon, \mathcal{F}_{m}\right\}_{m=0}^{\infty}, \varepsilon>0$ is a nonnegative one and satisfies the conditions

$$
\frac{f_{m}+\varepsilon}{f_{m-1}+\varepsilon} \leq \frac{D_{m}+\varepsilon}{\varepsilon}=C_{m}<\infty, m=\overline{1, \infty} .
$$

From Theorem 11 it follows the validity of the local regularity for the super-martingale $\left\{f_{m}+\varepsilon, \mathcal{F}_{m}\right\}_{m=0}^{\infty}$, therefore, for the super-martingale $\left\{f_{m}, \mathcal{F}_{m}\right\}_{m=0}^{\infty}$ the local regularity is also true.

\section{Local Regularity of Majorized Super-Martingales}

In this section, we give the elementary proof that a majorized super-martingale relative to the complete set of equivalent measures is local regular one.

Theorem 13. On a measurable space $\{\Omega, \mathcal{F}\}$ with a filtration $\mathcal{F}_{m}$ on it, let the set $M$ be a complete convex set of equivalent measures on $\mathcal{F}$ and the set $A_{0}$ contains an element $\xi_{0} \neq 1$. Then every bounded super-martingale $\left\{f_{m}, \mathcal{F}_{m}\right\}_{m=0}^{\infty}$ relative to the complete convex set of equivalent measures $M$ is a local regular one.

Proof. From Theorem 13 conditions, there exists a constant $0<C<\infty$ such that $\left|f_{m}\right| \leq C, m=\overline{1, \infty}$. Consider the super-martingale $\left\{f_{m}+C, \mathcal{F}_{m}\right\}_{m=0}^{\infty}$. Then $0 \leq f_{m}+C \leq 2 C$. Due to Consequence 1 , for the super-martingale $\left\{f_{m}+C, \mathcal{F}_{m}\right\}_{m=0}^{\infty}$ the local regularity is true. So, the same statement is valid for the super-martingale $\left\{f_{m}, \mathcal{F}_{m}\right\}_{m=0}^{\infty}$ Theorem 13 is proved.

The next Theorem is analogously proved as Theorem 13.

Theorem 14. On a measurable space $\{\Omega, \mathcal{F}\}$ with filtration $\mathcal{F}_{m}$ on it, let the set $\mathrm{M}$ be a complete convex set of equivalent measures on $\mathcal{F}$ and the set $A_{0}$ contains an element $\xi_{0} \neq 1$. Then a super-martingale $\left\{f_{m}, \mathcal{F}_{m}\right\}_{m=0}^{\infty}$ relative to the complete convex set of equivalent measures $M$ satisfying the conditions

$$
\left|f_{m}\right| \leq C_{1} \xi_{0}, \quad f_{m}+C_{1} \xi_{0} \leq C_{2}, \quad m=\overline{1, \infty}, \xi_{0} \in A_{0},
$$

for certain constants $0<C_{1}, C_{2}<\infty$ is a local regular one.

\section{Application to Mathematical Finance}

Due to Corollary 3, we can give the following definition of the fair price of contingent claim $f_{N}$ relative to a convex set of equivalent measures $M$. 
Definition 6. Let $f_{N}, N<\infty$ be a $\mathcal{F}_{N}$-measurable integrable random value relative to a convex set of equivalent measures $M$ such that for some $0 \leq \alpha_{0}<\infty$ and $\xi_{0} \in A_{0}$

$$
P\left(f_{N}-\alpha_{0} E^{P}\left\{\xi_{0} \mid \mathcal{F}_{N}\right\} \leq 0\right)=1 .
$$

Denote $G_{\alpha_{0}}=\left\{\alpha \in\left[0, \alpha_{0}\right], \exists \xi_{\alpha} \in A_{0}, P\left(f_{N}-\alpha E^{P}\left\{\xi_{\alpha} \mid \mathcal{F}_{N}\right\} \leq 0\right)=1\right\}$. We call

$$
f_{0}=\inf _{\alpha \in G_{\alpha_{0}}} \alpha
$$

the fair price of the contingent claim $f_{N}$ relative to a convex set of equivalent measures $M$, if there exists $\zeta_{0} \in A_{0}$ and a sequences $\alpha_{n} \in\left[0, \alpha_{0}\right], \quad \xi_{\alpha_{n}} \in A_{0}$, satisfying the conditions: $\alpha_{n} \rightarrow f_{0}, \xi_{\alpha_{n}} \rightarrow \zeta_{0}$ by probability, as $n \rightarrow \infty$, and such that

$$
P\left(f_{N}-\alpha_{n} E^{P}\left\{\xi_{\alpha_{n}} \mid \mathcal{F}_{N}\right\} \leq 0\right)=1, n=\overline{1, \infty} .
$$

Theorem 15. Let the set $A_{0}$ be uniformly integrable one relative to every measure $P \in M$. Suppose that for a nonnegative $\mathcal{F}_{N}$-measurable integrable contingent claim $f_{N}, N<\infty$ relative to every measure $P \in M$ there exist $\alpha_{0}<\infty$ and $\xi_{0} \in A_{0}$ such that

$$
P\left(f_{N}-\alpha_{0} E^{P}\left\{\xi_{0} \mid \mathcal{F}_{N}\right\} \leq 0\right)=1,
$$

then the fair price $f_{0}$ of contingent claim $f_{N}$ exists. For $f_{0}$ the inequality

$$
\sup _{P \in M} E^{P} f_{N} \leq f_{0}
$$

is valid. If $f_{N} \geq 0$ and a super-martingale $\left\{f_{m}=\underset{P \in M}{\operatorname{esssup}} E^{P}\left\{f_{N} \mid \mathcal{F}_{m}\right\}, \mathcal{F}_{m}\right\}_{m=0}^{\infty}$ is a local regular one, then $f_{0}=\sup _{P \in M} E^{P} f_{N}$.

Proof. If $f_{0}=\alpha_{0}$, then Theorem 15 is proved. Suppose that $f_{0}<\alpha_{0}$. Then there exists a sequence $\alpha_{n} \rightarrow f_{0}$, and $\xi_{\alpha_{n}} \in A_{0}, n \rightarrow \infty$, such that

$$
P\left(f_{N}-\alpha_{n} E^{P}\left\{\xi_{\alpha_{n}} \mid \mathcal{F}_{N}\right\} \leq 0\right)=1, P \in M .
$$

Due to the uniform integrability $A_{0}$ we obtain

$$
1=\lim _{n \rightarrow \infty} \int_{\Omega} \xi_{\alpha_{n}} \mathrm{~d} P=\int_{\Omega} \zeta_{0} \mathrm{~d} P, P \in M .
$$

Using again the uniform integrability of $A_{0}$ and going to the limit in (146) we obtain

$$
P\left(f_{N}-f_{0} E^{P}\left\{\zeta_{0} \mid \mathcal{F}_{N}\right\} \leq 0\right)=1, P \in M .
$$

From the inequality $f_{N}-f_{0} E^{P}\left\{\zeta_{0} \mid \mathcal{F}_{N}\right\} \leq 0$ it follows the inequality (145). If $f_{N} \geq 0$ and $\left\{f_{m}=\operatorname{esssup}_{P \in M} E^{P}\left\{f_{N} \mid \mathcal{F}_{m}\right\}, \mathcal{F}_{m}\right\}_{m=0}^{N}$ is a local regular super-martingale, then

$$
f_{m}=M_{m}-g_{m}, m=\overline{0, N}, g_{0}=0,
$$


where a martingale $\left\{M_{m}, \mathcal{F}_{m}\right\}_{m=0}^{N}$ is a nonnegative one and $E^{P} M_{m}=\sup _{P \in M} E^{P} f_{N}$ Introduce into consideration a random value $\xi_{0}=\frac{M_{N}}{\hat{f}_{0}}$, where $\hat{f}_{0}=\sup _{P \in M} E^{P} f_{N}$. Then $\xi_{0}$ belongs to the set $A_{0}$ and

$$
P\left(f_{N}-\hat{f}_{0} E^{P}\left\{\xi_{0} \mid \mathcal{F}_{N}\right\} \leq 0\right)=1 .
$$

From this it follows that $f_{0}=\sup E^{P} f_{N}$.

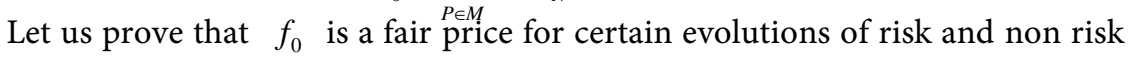
assets. Suppose that the evolution of risk asset is given by the law $S_{m}=f_{0} M^{P}\left\{\zeta_{0} \mid \mathcal{F}_{m}\right\}, m=\overline{0, N}$, and the evolution of non risk asset is given by the formula $B_{m}=1, m=\overline{0, N}$. lity

As proved above, for $f_{0}=\inf _{\alpha \in G_{\alpha_{0}}} \alpha$ there exists $\zeta_{0} \in A_{0}$ such that the inequa-

$$
f_{N}-f_{0} E^{P}\left\{\zeta_{0} \mid \mathcal{F}_{N}\right\} \leq 0
$$

is valid. Let us put

$$
\begin{aligned}
& f_{m}^{0}=f_{0} E^{P}\left\{\zeta_{0} \mid \mathcal{F}_{m}\right\}, P \in M, \\
& \bar{f}_{m}= \begin{cases}0, & m<N, \\
f_{N}-f_{0} E^{P}\left\{\zeta_{0} \mid \mathcal{F}_{m}\right\}, & m=N .\end{cases}
\end{aligned}
$$

It is evident that $\bar{f}_{m-1}-\bar{f}_{m} \geq 0, m=\overline{0, N}$. Therefore, the super-martingale

$$
f_{m}^{0}+\bar{f}_{m}= \begin{cases}f_{0} E^{P}\left\{\zeta_{0} \mid \mathcal{F}_{m}\right\}, & m<N, \\ f_{N}, & m=N,\end{cases}
$$

is a local regular one. It is evident that

$$
f_{m}^{0}+\bar{f}_{m}=M_{m}-g_{m}, m=\overline{0, N},
$$

where

$$
\begin{gathered}
M_{m}=f_{0} E^{P}\left\{\zeta_{0} \mid \mathcal{F}_{m}\right\}, m=\overline{0, N}, \\
g_{m}=0, m=\overline{0, N-1}, \\
g_{N}=f_{0} E^{P}\left\{\zeta_{0} \mid \mathcal{F}_{N}\right\}-f_{N} .
\end{gathered}
$$

For the martingale $\left\{M_{m}, \mathcal{F}_{m}\right\}_{m=0}^{N}$ the representation

$$
M_{m}=f_{0}+\sum_{i=1}^{m} H_{i} \Delta S_{i}, \quad m=\overline{0, N},
$$

is valid, where $H_{i}=1, i=\overline{1, N}$. Let us consider the trading strategy $\pi=\left\{\bar{H}_{m}^{0}, \bar{H}_{m}\right\}_{m=0}^{N}$, where

$$
\begin{gathered}
\bar{H}_{0}^{0}=f_{0}, \bar{H}_{m}^{0}=M_{m}-H_{m} S_{m}, m=\overline{1, N}, \\
\bar{H}_{0}=0, \bar{H}_{m}=H_{m}, m=\overline{1, N} .
\end{gathered}
$$

It is evident that $\bar{H}_{m}^{0}, \bar{H}_{m}$ are $\mathcal{F}_{m-1}$ measurable and the trading strategy $\pi$ satisfy self-financed condition

$$
\Delta \bar{H}_{m}^{0}+\Delta \bar{H}_{m} S_{m-1}=0 .
$$


Moreover, the capital corresponding to the self-financed trading strategy $\pi$ is given by the formula

$$
X_{m}^{\pi}=\bar{H}_{m}^{0}+\bar{H}_{m} S_{m}=M_{m} .
$$

Here from, $X_{0}^{\pi}=f_{0}$. Further,

$$
X_{N}^{\pi}=f_{N}+g_{N} \geq f_{N} .
$$

The last proves Theorem 15.

From (148) and Corollary 3 the Theorem 16 follows.

Theorem 16. Suppose that the set $A_{0}$ contains only $1 \leq k<\infty$ linear independent elements $\xi_{1}, \cdots, \xi_{k}$. If there exist $\xi_{0} \in T$ and $\alpha_{0} \geq 0$ such that

$$
P\left(f_{N}-\alpha_{0} E^{P}\left\{\xi_{0} \mid F_{N}\right\} \leq 0\right)=1, P \in M,
$$

where

$$
T=\left\{\xi \geq 0, \xi=\sum_{i=1}^{k} \alpha_{i} \xi_{i}, \alpha_{i} \geq 0, i=\overline{1, k}, \sum_{i=1}^{k} \alpha_{i}=1\right\}
$$

then the fair price $f_{0}$ of the contingent claim $f_{N} \geq 0$ exists, where $f_{N}$ is $F_{N}$ measurable and integrable relative to every measure $P \in M, N<\infty$.

Proof. The proof is evident, as the set $T$ is a uniformly integrable one relative to every measure from $M$.

Corollary 4. On a measurable space $\{\Omega, \mathcal{F}\}$ with filtration $\mathcal{F}_{m}$ on it, let $\left\{f_{m}, \mathcal{F}_{m}\right\}_{m=0}^{N}$ be a non negative local regular super-martingale relative to a convex set of equivalent measures $M$. If the set $A_{0}$ is uniformly integrable relative to every measure $P \in M$, then the fair price of contingent claim $f_{N}$ exists.

Proof. From the local regularity of super-martingale $\left\{f_{m}, \mathcal{F}_{m}\right\}_{m=0}^{N}$ we have $f_{m}=M_{m}-g_{m}, m=\overline{0, N}$. Therefore, $P\left(f_{N}-\alpha_{0} \xi_{0} \leq 0\right)=1$, where $\alpha_{0}=E^{P} M_{N}, P \in M, \xi_{0}=\frac{M_{N}}{E^{P} M_{N}}$. From the last it follows that the conditions of Theorem 15 are satisfied. Corollary 4 is proved.

On a probability space $\{\Omega, \mathcal{F}, P\}$, let us consider an evolution of one risk asset given by the law $\left\{S_{m}\right\}_{m=0}^{N}$, where $S_{m}$ is a random value taking values in $R_{+}^{1}$. Suppose that $\mathcal{F}_{m}$ is a filtration on $\{\Omega, \mathcal{F}, P\}$ and $S_{m}$ is $\mathcal{F}_{m}$-measurable random value. We assume that the non risk asset evolve by the law $B_{m}^{0}=1, m=\overline{1, N}$. Denote $M^{e}(S)$ the set of all martingale measures being equivalent to the measure $P$. We assume that the set $M^{e}(S)$ of such martingale measures is not empty and the effective market is non complete, see, for example, [16] [17] [18] [19]. So, we have that

$$
E^{Q}\left\{S_{m} \mid \mathcal{F}_{m-1}\right\}=S_{m-1}, m=\overline{1, N}, Q \in M^{e}(S) .
$$

The next Theorem justifies the Definition 6.

Theorem 17. Let a contingent claim $f_{N}$ be a $\mathcal{F}_{N}$-measurable integrable random value with respect to every measure from $M^{e}(S)$ and the conditions 
of the Theorem 16 are satisfied with $\xi_{i}=\frac{S_{i}}{S_{0}}, i=\overline{0, N}$. Then there exists self-financed trading strategy $\pi$ the capital evolution $\left\{X_{m}^{\pi}\right\}_{m=0}^{N}$ of which is a martingale relative to every measure from $M^{e}(S)$ satisfying conditions $X_{0}^{\pi}=f_{0}, X_{N}^{\pi} \geq f_{N}$, where $f_{0}$ is a fair price of contingent claim $f_{N}$.

Proof. Due to Theorems 15, 16, for $f_{0}=\inf _{\alpha \in G_{\alpha_{0}}} \alpha$ there exists $\zeta_{0} \in A_{0}$ such that the inequality

$$
f_{N}-f_{0} E^{P}\left\{\zeta_{0} \mid \mathcal{F}_{N}\right\} \leq 0
$$

is valid. Let us put

$$
\begin{gathered}
f_{m}^{0}=f_{0} E^{P}\left\{\zeta_{0} \mid \mathcal{F}_{m}\right\}, P \in M^{e}(S), \\
\bar{f}_{m}= \begin{cases}0, & m<N, \\
f_{N}-f_{0} E^{P}\left\{\zeta_{0} \mid \mathcal{F}_{m}\right\}, & m=N .\end{cases}
\end{gathered}
$$

It is evident that $\bar{f}_{m-1}-\bar{f}_{m} \geq 0, m=\overline{0, N}$. Therefore, the super-martingale

$$
f_{m}^{0}+\bar{f}_{m}= \begin{cases}f_{0} E^{P}\left\{\zeta_{0} \mid \mathcal{F}_{m}\right\}, & m<N \\ f_{N}, & m=N\end{cases}
$$

is a local regular one. It is evident that

$$
f_{m}^{0}+\bar{f}_{m}=M_{m}-g_{m}, m=\overline{0, N},
$$

where

$$
\begin{gathered}
M_{m}=f_{0} E^{P}\left\{\zeta_{0} \mid \mathcal{F}_{m}\right\}, m=\overline{0, N}, \\
g_{m}=0, m=\overline{0, N-1,} \\
g_{N}=f_{0} E^{P}\left\{\zeta_{0} \mid \mathcal{F}_{N}\right\}-f_{N} .
\end{gathered}
$$

Due to Theorem 20, for the martingale $\left\{M_{m}\right\}_{m=0}^{N}$ the representation

$$
M_{m}=f_{0}+\sum_{i=1}^{m} H_{i} \Delta S_{i}, m=\overline{0, N},
$$

is valid. Let us consider the trading strategy $\pi=\left\{\bar{H}_{m}^{0}, \bar{H}_{m}\right\}_{m=0}^{N}$, where

$$
\begin{gathered}
\bar{H}_{0}^{0}=f_{0}, \bar{H}_{m}^{0}=M_{m}-H_{m} S_{m}, m=\overline{1, N}, \\
\bar{H}_{0}=0, \bar{H}_{m}=H_{m}, \quad m=\overline{1, N} .
\end{gathered}
$$

It is evident that $\bar{H}_{m}^{0}, \bar{H}_{m}$ are $\mathcal{F}_{m-1}$-measurable ones and the trading strategy $\pi$ satisfy the self-financed condition

$$
\Delta \bar{H}_{m}^{0}+\Delta \bar{H}_{m} S_{m-1}=0
$$

Moreover, a capital corresponding to the self-financed trading strategy $\pi$ is given by the formula

$$
X_{m}^{\pi}=\bar{H}_{m}^{0}+\bar{H}_{m} S_{m}=M_{m} .
$$

Herefrom, $X_{0}^{\pi}=f_{0}$. Further,

$$
X_{N}^{\pi}=f_{N}+g_{N} .
$$


Therefore $X_{N}^{\pi} \geq f_{N}$. Theorem 17 is proved.

In the next Theorem we assume that the evolutions of risk and non risk assets generate incomplete market [16] [17] [18] [19] [20], that is, the set of martingale measures contains more that one element.

Theorem 18. Let an evolution $\left\{S_{m}\right\}_{m=0}^{N}$ of the risk asset satisfy the conditions $P\left(D_{m}^{1} \leq S_{m} \leq D_{m}^{2}\right)=1$, where the constants $D_{m}^{i}$ satisfy the inequalities $D_{m-1}^{1} \geq D_{m}^{1}>0, \quad D_{m-1}^{2} \leq D_{m}^{2}<\infty, m=\overline{1, N}$, and let the non risk asset evolution be deterministic one given by the law $\left\{B_{m}\right\}_{m=0}^{N}, B_{m}=1, m=\overline{0, N}$. The fair price of Standard European Call Option with the payment function $f_{N}=\left(S_{N}-K\right)^{+}$is given by the formula

$$
f_{0}= \begin{cases}S_{0}\left(1-\frac{K}{D_{N}^{2}}\right), & K \leq D_{N}^{2}, \\ 0, & K>D_{N}^{2} .\end{cases}
$$

The fair price of Standard European Put Option with the payment function $f_{N}=\left(K-S_{N}\right)^{+}$is given by the formula

$$
f_{0}= \begin{cases}K-D_{N}^{1}, & K \geq D_{N}^{1}, \\ 0, & K<D_{N}^{1} .\end{cases}
$$

Proof. In Theorem 18 conditions, the set of equations $E^{P} \zeta=1, \zeta \geq 0$ has the solutions $\zeta_{i}=\frac{S_{i}}{S_{0}}, i=\overline{0, N}$. It is evident that $\alpha_{0}=S_{0}$ and $\zeta_{N}=\frac{S_{N}}{S_{0}}$, since

$$
\frac{\left(S_{N}-K\right)^{+}}{B_{N}}-\alpha_{0} \frac{S_{N}}{S_{0}} \leq 0, \omega \in \Omega .
$$

Let us prove the needed formula. Consider the inequality

$$
\left(S_{N}-K\right)-\alpha \sum_{i=0}^{N} \gamma_{i} \frac{S_{i}}{S_{0}} \leq 0, \gamma \in V_{0}
$$

where $V_{0}=\left\{\gamma=\left\{\gamma_{i}\right\}_{i=0}^{N}, \gamma_{i} \geq 0, \sum_{i=0}^{N} \gamma_{i}=1\right\}$. Or,

$$
S_{N}\left(1-\frac{\alpha \gamma_{N}}{S_{0}}\right)-K-\alpha \sum_{i=0}^{N-1} \gamma_{i} \frac{S_{i}}{S_{0}} \leq 0 .
$$

Suppose that $\alpha$ satisfies the inequality

$$
1-\frac{\alpha}{S_{0}}>0
$$

If $\alpha$ satisfies additionally the equality

$$
D_{N}^{2}\left(1-\frac{\alpha \gamma_{N}}{S_{0}}\right)-K-\alpha \sum_{i=0}^{N-1} \gamma_{i} \frac{D_{i}^{1}}{S_{0}}=0,
$$

then for all $\omega \in \Omega$ (186) is valid. From (188) we obtain for $\alpha$

$$
\alpha=\frac{S_{0}\left(D_{N}^{2}-K\right)}{\left(D_{N}^{2} \gamma_{N}+\sum_{i=0}^{N-1} \gamma_{i} D_{i}^{1}\right)} .
$$


If $D_{N}^{2}-K>0$, then

$$
\inf _{\gamma \in V_{0}} \frac{S_{0}\left(D_{N}^{2}-K\right)}{\left(D_{N}^{2} \gamma_{N}+\sum_{i=0}^{N-1} \gamma_{i} D_{i}^{1}\right)}=\frac{S_{0}\left(D_{N}^{2}-K\right)}{D_{N}^{2}},
$$

since $D_{N}^{2} \geq D_{i}^{1}$. From here we obtain

$$
f_{0}=S_{0}\left(1-\frac{K}{D_{N}^{2}}\right) .
$$

It is evident that $\alpha=f_{0}$ satisfies the inequality (187).

If $D_{N}^{2}-K \leq 0$, then $S_{N}-K \leq 0$ and from (185) we can put $\alpha=0$. Then, the formula (186) is valid for all $\omega \in \Omega$.

Let us prove the formula (183) for Standard European Put Option. If $S_{N} \leq K$ it is evident that $\alpha_{0}=K$, and $\zeta_{0}=1$, since

$$
\left(K-S_{N}\right)-\alpha_{0} \leq 0, \omega \in \Omega .
$$

Let us prove the needed formula. Consider the inequality

$$
\left(K-S_{N}\right)^{+}-\alpha \sum_{i=0}^{N} \gamma_{i} \frac{S_{i}}{S_{0}} \leq 0, \gamma \in V_{0} .
$$

Or, for $S_{N} \leq K$

$$
-S_{N}\left(1+\frac{\alpha \gamma_{N}}{S_{0}}\right)+K-\alpha \sum_{i=0}^{N-1} \gamma_{i} \frac{S_{i}}{S_{0}} \leq 0
$$

If $\alpha$ is a solution of the equality

$$
-D_{N}^{1}\left(1+\frac{\alpha \gamma_{N}}{S_{0}}\right)+K-\alpha \sum_{i=0}^{N-1} \gamma_{i} \frac{D_{i}^{1}}{S_{0}}=0,
$$

then for all $\omega \in \Omega \quad(194)$ is valid. From (195) we obtain for $\alpha$

$$
\alpha=\frac{S_{0}\left(K-D_{N}^{1}\right)}{\sum_{i=0}^{N} \gamma_{i} D_{i}^{1}} .
$$

Therefore,

$$
\inf _{\gamma \in V_{0}} \frac{S_{0}\left(K-D_{N}^{1}\right)}{\sum_{i=0}^{N} \gamma_{i} D_{i}^{1}}=K-D_{N}^{1},
$$

since $D_{i}^{1} \leq S_{0}, i=\overline{1, N}, D_{0}^{1}=S_{0}$. From here we obtain

$$
f_{0}=K-D_{N}^{1} \text {. }
$$

If $D_{N}^{1}-K>0$, then $S_{N}-K>0$ and from (193) we can put $\alpha=0$. Then, (194) is valid for all $\omega \in \Omega$. The Theorem 18 is proved.

\section{Some Auxiliary Results}

On a measurable space $\{\Omega, \mathcal{F}\}$ with filtration $\mathcal{F}_{n}$ on it, let us consider a convex set of equivalent measures $M$. Suppose that $\xi_{1}, \cdots, \xi_{d}$ is a set of random 
values belonging to the set $A_{0}$. Introduce $d$ martingales relative to a set of measures $M\left\{S_{n}^{i}, \mathcal{F}_{n}\right\}_{n=0}^{\infty}, i=\overline{1, d}$, where $S_{n}^{i}=E^{P}\left\{\xi_{i} \mid \mathcal{F}_{n}\right\}, i=\overline{1, d}, P \in M$. Denote by $M^{e}(S)$ a set of all martingale measures equivalent to a measure $P \in M$, that is, $Q \in M^{e}(S)$ if

$$
E^{Q}\left\{S_{n} \mid \mathcal{F}_{n-1}\right\}=S_{n-1}, E^{Q}\left|S_{n}\right|<\infty, Q \in M^{e}(S), n=\overline{1, \infty} .
$$

It is evident that $M \subseteq M^{e}(S)$ and $M^{e}(S)$ is a convex set. Denote $P_{0}$ a certain fixed measure from $M^{e}(S)$ and let $L^{0}\left(R^{d}\right)$ be a set of finite valued random values on a probability space $\left\{\Omega, \mathcal{F}, P_{0}\right\}$, taking values in $R^{d}$.

Let $H^{0}$ be a set of finite valued predictable processes $H=\left\{H_{n}\right\}_{n=1}^{N}$, where $H_{n}=\left\{H_{n}^{i}\right\}_{i=1}^{d}$ takes values in $R^{d}$ and $H_{n}$ is $\mathcal{F}_{n-1}$-measurable random vector. Introduce into consideration a set of random values

$$
\begin{gathered}
K_{N}^{1}=\left\{\xi \in L^{0}\left(R^{1}\right), \xi=\sum_{k=1}^{N}\left\langle H_{k}, \Delta S_{k}\right\rangle,, H \in H^{0}\right\}, N<\infty, \\
\Delta S_{k}=S_{k}-S_{k-1},\left\langle H_{k}, \Delta S_{k}\right\rangle=\sum_{s=1}^{d} H_{k}^{s}\left(S_{k}^{s}-S_{k-1}^{s}\right) .
\end{gathered}
$$

Lemma 13. The set of random values $K_{N}^{1}$ is a closed subset in the set of finite valued random values $L^{0}\left(R^{1}\right)$ relative to the convergence by measure $P \in M$.

The proof of the Lemma 13 see, for example, [17].

Introduce into consideration a subset

$$
V^{0}=\left\{H \in H^{0},\left\|H_{n}\right\|<\infty, n=\overline{1, N}\right\}
$$

of the set $H^{0}$, where $\left\|H_{n}\right\|=\sup _{\omega \in \Omega} \sum_{i=1}^{d}\left|H_{n}^{i}\right|$. Let $K_{N}$ be a subset of the set $K_{N}^{1}$

$$
K_{N}=\left\{\xi \in L^{0}\left(R^{1}\right), \xi=\sum_{k=1}^{N}\left\langle H_{k}, \Delta S_{k}\right\rangle, H \in V^{0}\right\} .
$$

Denote also a set

$$
C=\left\{k-f, k \in K_{N}, f \in L_{+}^{\infty}\left(\Omega, \mathcal{F}, P_{0}\right)\right\},
$$

where $L_{+}^{\infty}\left(\Omega, \mathcal{F}, P_{0}\right)$ is a set of bounded nonnegative random values. Let $\bar{C}$ be the closure of $C$ in $L^{1}\left(\Omega, \mathcal{F}, P_{0}\right)$ metrics.

Lemma 14. If $\zeta \in \bar{C}$ and such that $E^{P_{0}} \zeta=0$, then for $\zeta$ the representation

$$
\zeta=\sum_{k=1}^{N}\left\langle H_{k}, \Delta S_{k}\right\rangle
$$

is valid for a certain finite valued predictable process $H=\left\{H_{n}\right\}_{n=1}^{N}$.

Proof. If $\zeta \in K_{N}$, then Lemma 14 is proved. Suppose that $\zeta \in \bar{C}$, then there exists a sequence $k_{n}-f_{n}, k_{n} \in K_{N}, f_{n} \in L_{+}^{\infty}\left(\Omega, \mathcal{F}, P_{0}\right)$ such that $\left\|k_{n}-f_{n}-\zeta\right\|_{P_{0}} \rightarrow 0, n \rightarrow \infty$, where $\|g\|_{P_{0}}=E^{P_{0}}|g|$. Since $\left|E^{P_{0}}\left(k_{n}-f_{n}-\zeta\right)\right| \leq\left\|k_{n}-f_{n}-\zeta\right\|_{P_{0}}$, we have $E^{P_{0}} f_{n} \leq\left\|k_{n}-f_{n}-\zeta\right\|_{P_{0}}$. From here 
we obtain $\left\|k_{n}-\zeta\right\|_{P_{0}} \leq 2\left\|k_{n}-f_{n}-\zeta\right\|_{P_{0}}$. Therefore, $k_{n} \rightarrow \zeta$ by measure $P_{0}$. On the basis of Lemma 13, a set

$$
\begin{gathered}
K_{N}^{1}=\left\{\xi \in L^{0}\left(R^{1}\right), \xi=\sum_{k=1}^{N}\left\langle H_{k}, \Delta S_{k}\right\rangle, H \in H^{0}\right\}, \\
\left\langle H_{k}, \Delta S_{k}\right\rangle=\sum_{i=1}^{d} H_{k}^{i}\left(S_{k}^{i}-S_{k-1}^{i}\right)
\end{gathered}
$$

is a closed subset of $L^{0}\left(R^{1}\right)$ relative to the convergence by measure $P_{0}$. From this fact, we obtain the proof of Lemma 14, since there exists the finite valued predictable process $H \in H^{0}$ such that for $\zeta$ the representation

$$
\zeta=\sum_{k=1}^{N}\left\langle H_{k}, \Delta S_{k}\right\rangle
$$

is valid.

Theorem 19 Let $E^{Q}|\zeta|<\infty, Q \in M^{e}(S)$. If for every $Q \in M^{e}(S), E^{Q} \zeta=0$, then there exists finite valued predictable process $H$ such that for $\zeta$ the representation

$$
\zeta=\sum_{k=1}^{N}\left\langle H_{k}, \Delta S_{k}\right\rangle
$$

is valid.

Proof. If $\zeta \in \bar{C}$, then (209) follows from Lemma 14. So, let $\zeta$ does not belong to $\bar{C}$. As in Lemma $14, \bar{C}$ is a closure of $C$ in $L^{1}\left(\Omega, \mathcal{F}, P_{0}\right)$ metrics for the fixed measure $P_{0}$. The set $\bar{C}$ is a closed convex set in $L^{1}\left(\Omega, \mathcal{F}, P_{0}\right)$. Consider the other convex closed set that consists from one element $\zeta$. Due to Han-Banach Theorem, there exists a linear continuous functional $l_{1}$, which belongs to $L^{\infty}\left(\Omega, \mathcal{F}, P_{0}\right)$, and real numbers $\alpha>\beta$ such that

$$
l_{1}(\xi)=\int_{\Omega} \xi(\omega) q(\omega) \mathrm{d} P_{0}, q(\omega) \in L^{\infty}\left(\Omega, \mathcal{F}, P_{0}\right),
$$

and the inequalities $l_{1}(\zeta)>\alpha, l_{1}(\xi) \leq \beta, \xi \in \bar{C}$ are valid. Since $\bar{C}$ is a convex cone we can put $\beta=0$. From the condition $l_{1}(\xi) \leq 0, \xi \in \bar{C}$ we have $l_{1}(\xi)=0, \xi \in K_{N}^{1} \cap L^{1}\left(\Omega, \mathcal{F}, P_{0}\right)$. From (210) and the inclusions $\bar{C} \supset C \supset-L^{\infty}\left(\Omega, \mathcal{F}, P_{0}\right)$ we have $q(\omega) \geq 0$. Introduce a measure

$$
Q^{*}(A)=\int_{A} q(\omega) \mathrm{d} P_{0}\left[\int_{\Omega} q(\omega) \mathrm{d} P_{0}\right]^{-1} .
$$

Then, we have

$$
\int_{\Omega} \xi(\omega) \mathrm{d} Q^{*}=0, \xi \in K_{N}^{1} \cap L^{1}\left(\Omega, \mathcal{F}, P_{0}\right) .
$$

Let us choose $\xi=\chi_{A}(\omega)\left(S_{i}^{j}-S_{i-1}^{j}\right), A \in \mathcal{F}_{i-1}$, where $\chi_{A}(\omega)$ is an indicator of a set $A$. We obtain

$$
\int_{A}\left(S_{i}^{j}-S_{i-1}^{j}\right) \mathrm{d} Q^{*}=0, A \in \mathcal{F}_{i-1} .
$$

So, $Q^{*}$ is a martingale measure that belongs to the set $M^{a}(S)$, which is a 
set of absolutely continuous martingale measures. Let us choose $Q \in M^{e}(S)$ and consider a measure $Q_{1}=(1-\gamma) Q+\gamma Q^{*}, 0<\gamma<1$. A measure $Q_{1} \in M^{e}(S)$ and, moreover, $E^{Q_{1}} \zeta=\gamma E^{Q^{*}} \zeta>0$. We come to the contradiction with the conditions of Theorem 19, since for $Q \in M^{e}(S), E^{Q} \zeta=0$. So, $\zeta \in \bar{C}$, and in accordance with Lemma 14 , for $\zeta$ the declared representation in Theorem 19 is valid.

Theorem 20. For every martingale $\left\{M_{n}, \mathcal{F}_{n}\right\}_{n=0}^{\infty}$ relative to the set of measures $M^{e}(S)$, there exists a predictable random process $H$ such that for $M_{n}, n=\overline{0, \infty}$, the representation

$$
M_{n}=M_{0}+\sum_{i=1}^{n}\left\langle H_{i}, \Delta S_{i}\right\rangle, \quad n=\overline{1, \infty}
$$

is valid.

Proof. For fixed natural $N \geq 1$, let us consider the random value $M_{N}-M_{0}=\zeta$. Since

$$
E^{Q}|\zeta|<\infty, E^{Q} \zeta=0, Q \in M^{e}(S)
$$

then $\zeta$ satisfies the conditions of Theorem 19 and, therefore, belongs to $\bar{C}$, so, there exists a sequence $k_{n}=\sum_{i=1}^{N}\left\langle H_{i}^{n}, \Delta S_{i}\right\rangle \in K_{N}$ such that

$$
\int_{\Omega}\left|k_{n}-\zeta\right| \mathrm{d} P_{0} \rightarrow 0, n \rightarrow \infty
$$

From here, we obtain

$$
\int_{\Omega}\left|E^{P_{0}}\left\{\left(k_{n}-\zeta\right) \mid \mathcal{F}_{m}\right\}\right| \mathrm{d} P_{0} \leq \int_{\Omega}\left|k_{n}-\zeta\right| \mathrm{d} P_{0} \rightarrow 0, n \rightarrow \infty
$$

But $E^{P_{0}}\left\{k_{n} \mid \mathcal{F}_{m}\right\}=\sum_{i=1}^{m}\left\langle H_{i}^{n}, \Delta S_{i}\right\rangle$. Hence, we obtain that both $\sum_{i=1}^{m}\left\langle H_{i}^{n}, \Delta S_{i}\right\rangle$ and $\sum_{i=1}^{N}\left\langle H_{i}^{n}, \Delta S_{i}\right\rangle$ converges by measure $P_{0}$ to $E^{P_{0}}\left\{\zeta \mid \mathcal{F}_{m}\right\}$ and $\zeta$, correspondingly. There exists a subsequence $n_{k}$ such that $H^{n_{k}}$ converges everywhere to predictable process $H$. From here, we have $\zeta=\sum_{i=1}^{N}\left\langle H_{i}, \Delta S_{i}\right\rangle$ and $E^{P_{0}}\left\{\zeta \mid \mathcal{F}_{m}\right\}=\sum_{i=1}^{m}\left\langle H_{i}, \Delta S_{i}\right\rangle$. It proves that for all $m<N$

$$
M_{m}=M_{0}+\sum_{i=1}^{m}\left\langle H_{i}, \Delta S_{i}\right\rangle
$$

Theorem 20 is proved.

\section{Conclusions}

In the paper, we generalize Doob decomposition for super-martingales relative to one measure onto the case of super-martingales relative to a convex set of equivalent measures. For super-martingales relative to one measure for continuous time Doob's result was generalized in papers [21] [22]. Section 2 contains the definition of local regular super-martingales. Theorem 1 gives the necessary 
and sufficient conditions of the local regularity of super-martingale. In spite of its simplicity, the Theorem 1 appeared very useful for the description of the local regular super-martingales.

For this purpose we investigate the structure of super-martingales of special types relative to the convex set of equivalent measures, generated by a certain finite set of equivalent measures. The main result of Section 3 is Lemma 6, which allowed proving Lemma 8, giving the sufficient conditions of the existence of a martingale with respect to a convex set of equivalent measures generated by finite set of equivalent measures.

Theorem 2 describes all local regular non-negative super-martingales of the special type (30) relative to the convex set of equivalent measures, generated by the finite set of equivalent measures.

In the Theorem 3, we give the sufficient conditions of the existence of the local regular martingale relative to an arbitrary set of equivalent measures and arbitrary filtration. After that, we present in Theorem 4 the important construction of the local regular super-martingales which we sum up in Corollary 2. Theorem 6 proves that every majorized super-martingale belongs to the described class (53) of the local regular super-martingales.

Theorem 7 gives a variant of the necessary and sufficient conditions of local regularity of non-negative super-martingale relative to a convex set of equivalent measures. Definition 3 determines a class of the complete set of equivalent measures. Lemma 10 guarantees a bound (77) for all non-negative random values allowing us to prove Theorem 8 , stating that for every super-martingale the optional decomposition is valid. We extend the results obtained from the finite space of elementary events onto the case as a space of elementary events is a countable one. At last, the subsection 5.3 contains the generalization of the result obtained in subsection 5.2 onto the case of arbitrary space of elementary events. In Section 6, we prove Theorems 13 and 14, stating that for every majorized super-martingale the optional decomposition is valid.

Corollary 3 contains the important construction of the local regular super-martingales playing the important role in the definition of the fair price of contingent claim relative to a convex set of equivalent measures. The Definition 6 is a fundamental one for the evaluation of risks in incomplete markets. Theorem 15 gives the sufficient conditions of the existence of the fair price of contingent claim relative to a convex set of equivalent measures. It also gives the sufficient conditions, when the defined fair price coincides with the classical value. In Theorem 16 the simple conditions of the existence of the fair price of contingent claim are given. In Theorem 17 we prove the existence of the self-financed trading strategy confirming the Definition 6 of the fair price as the parity between the long and short positions in contracts. As an application of the results obtained we prove Theorem 18, where the formulas for the Standard European Call and Put Options in an incomplete market we present. Section 8 contains auxiliary results needed for previous sections. 


\section{References}

[1] Kramkov, D.O. (1996) Optional Decomposition of Super-Martingales and Hedging in Incomplete Security Markets. Probability Theory and Related Fields, 105, 459-479. https://doi.org/10.1007/BF01191909

[2] Follmer, H. and Kramkov, D.O. (1997) Optional Decomposition Theorem under Constraints. Probability Theory and Related Fields, 109, 1-25. https://doi.org/10.1007/s004400050122

[3] Follmer, H. and Kabanov, Yu.M. (1996) Optional Decomposition Theorems in Discrete Time. Atti del convegno in onore di Oliviero Lessi, Padova, 25-26 marzo 1996, 47-68.

[4] Follmer, H. and Kabanov, Yu.M. (1998) Optional Decomposition and Lagrange Multipliers. Finance and Stochastics, 2, 69-81.

[5] El Karoui, N. and Quenez, M.C. (1995) Dynamic Programming and Pricing of Contingent Claims in an Incomplete Market. SIAM Journal on Control and Optimization, 33, 27-66. https://doi.org/10.1137/S0363012992232579

[6] Bouchard, B. and Nutz, M. (2015) Arbitrage and Duality in Nondominated Discrete-Time Models. The Annals of Applied Probability, 25, 823-859. https://doi.org/10.1214/14-AAP1011

[7] Gonchar, N.S. (2008) Mathematical Foundations of Information Economics. Bogolyubov Institute for Theoretical Physics, Kiev.

[8] Gonchar, N.S. (2015) Mathematical Model of Banking Operation. Cybernetics and System Analysis, 51, 378-399. https://doi.org/10.1007/s10559-015-9730-0

[9] Gonchar, N.S. and Terentieva, L.S. (2008) Default Risk Valuation of the Firm with the Special Process of Internal Yield. Journal of Automation and Information Sciences, 40, 57-71. https://doi.org/10.1615/JAutomatInfScien.v40.i8.60

[10] Gonchar, N.S. (2017) Banking and Risk Assessment, Chapter 8. In: Banking. Services, Opportunity and Risks, Nova Science Publisher, Inc., New York.

[11] Gonchar, N.S. (2001) Stock Market and Economic Growth. Oberehy, Kiev. (In Ukrainian)

[12] Kallianpur, G. (1980) Stochastic Filtering Theory. Springer, New York. https://doi.org/10.1007/978-1-4757-6592-2

[13] Chow, Y.S., Robbins, H. and Siegmund, D. (1971) Great Expectations: The Theory of Optimal Stopping. Houghton Mifflin Company, Boston.

[14] Kelley, J. (1955) General Topology. Van Nostrand, New York.

[15] Delbaen, F. and Schachermayer, W. (2006) The Mathematics and Arbitrage. Springer, Berlin.

[16] Delbaen, F. and Schachermaer, W. (1994) A General Version of Fundamental Theorem of Asset Pricing. Mathematische Annalen, 300, 463-520. https://doi.org/10.1007/BF01450498

[17] Dalang, R.C., Morton, A. and Willinger, W. (1990) Equivalent Martingale Measures and No-Arbitrage in Stochastic Securities Market Model. Stochastics and Stochastic Reports, 29, 185-201. https://doi.org/10.1080/17442509008833613

[18] Kreps, D.M. (1981) Arbitrage and Equilibrium in Economics with Infinitely Many Commodities. Journal of Mathematical Economics, 8, 15-35.

https://doi.org/10.1016/0304-4068(81)90010-0

[19] Harrison, J.M. and Kreps, D.M. (1979) Martingales and Arbitrage in Multiperiod Securities Markets. Journal of Economic Theory, 20, 381-408. 
https://doi.org/10.1016/0022-0531(79)90043-7

[20] Harrison, J.M. and Pliska, S.R. (1981) Martingales and Stochastic Integrals in the Theory of Continuous Trading. Stochastic Processes and their Applications, 11, 215-260. https://doi.org/10.1016/0304-4149(81)90026-0

[21] Meyer, P.A. (1963) A Decomposition Theorem for Super-Martingales. Illinois Journal of Mathematics, 7, 1-17.

[22] Meyer, P.A. (1972) A Decomposition for Super-Martingales: The Uniqueness Theorem. Illinois Journal of Mathematics, 6, 193-205. 